\title{
Modeling and Analysis of the Composite Stiffness for Angular Contact Ball Bearings
}

\author{
Jianhua Li, Chunli Lei $\mathbb{i}$, Baoru Gong, Pan Cui, and Xibin Jia \\ School of Mechanical and Electrical Engineering, Lanzhou University of Technology, Lanzhou 730050, China \\ Correspondence should be addressed to Chunli Lei; 1clyq2004@163.com
}

Received 26 June 2020; Revised 18 October 2020; Accepted 23 October 2020; Published 6 November 2020

Academic Editor: Trupti Ranjan Lenka

Copyright (c) 2020 Jianhua Li et al. This is an open access article distributed under the Creative Commons Attribution License, which permits unrestricted use, distribution, and reproduction in any medium, provided the original work is properly cited.

\begin{abstract}
As a core component of the motorized spindle, the dynamic stiffness of the angular contact ball bearing directly affects the dynamic characteristics of machinery. A modified quasistatic model of the ball bearing is established considering the influences of thermal deformation, centrifugal deformation, and elastohydrodynamic lubrication (EHL). Then, the film stiffness model considering spin motion is constructed. On this basis, the composite stiffness model of the ball bearing is proposed, and the effects of different factors on dynamic characteristic parameters are investigated. The results show that different factors have different effects on the dynamic parameters. With the increase in preload, the contact stiffness and composite stiffness increase. Considering EHL, the radial contact stiffness and composite stiffness increase while the axial and angular contact stiffness and composite stiffness decrease. Considering the thermal effect and centrifugal effect, the radial contact stiffness and composite stiffness increase while the axial and angular contact stiffness and composite stiffness decrease. The film stiffness and composite stiffness increase with the consideration of the spinning motion.
\end{abstract}

\section{Introduction}

With the development of science and technology, the level of modern manufacturing technology and CNC processing technology has been improved sharply. The motorized spindle is the kernel component of machine tools. The bearing is the support component of the motorized spindle, and its performance directly affects the processing precision and efficiency of the motorized spindle and the whole machine tool. The stiffness of the bearing has an important influence on the dynamic performance of the rotor-bearing system and also directly affects the machining precision [1]. The dynamic characteristic parameters of the bearing are important indexes to evaluate the performance of the bearing and the factors to be considered in bearing design. Therefore, the dynamic characteristics of the angular contact ball bearing are studied, which not only improves the performance analysis level of the bearing but also provides a new design idea for modern manufacturing industry.

Numerous simulation and modeling methods were reported to analyze the dynamic characteristics of the rolling bearing. For instance, Jones [2] introduced the inertia effect into the balance equation of the bearing and established the quasistatic mechanical model of the bearing force analysis. Harris [3] developed and improved Jones' method and added the influence of EHL to establish the quasidynamic model of the bearing. After that, Gupta [4] considered the motion and stress state of the rolling element and the interaction between the parts and put forward the dynamic model of the rolling bearing. Li and Sun [5] studied the bearing stiffness but ignored the role of oil film. Noel et al. [6] established a new stiffness matrix calculation method based on the dynamic model of the rolling bearing. Liu et al. [7] obtained the load distribution and stiffness of the angular contact ball bearing under the condition of combined load using a new analytical method. Bizarre et al. [8] established the stiffness model of the angular contact ball bearing and evaluated the equivalent parameters of stiffness and damping of each contact point under different loading conditions. Guo et al. [9] presented the EHL model with spin motion and calculated the dynamic characteristics of the angular contact ball bearing. Lei et al. [10] constructed a 
novel film stiffness model of the rolling bearing, which could consider the non-Newton fluid EHL, rough surface morphology, thermal effect, and time-dependent effect, and the numerical simulation was carried out to analyze the influence of different parameters on the film stiffness. Zhang et al. [11] established a novel quasistatic five-degree-of-freedom model for the angular contact ball bearing of the spindle under rigid axial preload and employed a local bearing heatgeneration power model to assess power losses in different contact zones. Liu [12] conducted a new analytical dynamic model for a rotor-roller bearing-housing systems (RBHSs), which could consider the time-dependent additional contact zone excitation caused by the fault on the raceways, deformable interface between the outer raceway and housing, lubricating oil film, and deformable rotor and housing. Liu and Shao [13] proposed a new 12-DOF dynamic model for rigid rotor-bearing systems (RBSs), which could formulate the housing support stiffness, interfacial frictional moments including load dependent and load independent components, time-varying displacement excitation caused by a LOD, additional deformations at the sharp edges of the LOD, and lubricating oil film. Based on the Hertz contact theory, EHL model, and Jones' bearing theory, Lei et al. [14] proposed the comprehensive stiffness model of the angular contact ball bearing in consideration of the effects of elastic deformation, centrifugal deformation, thermal deformation, and the ball spinning motion. Wang and Zhang [15] presented a derivation of the lubrication equation suitable for the nonlinear rheological model. Full numerical solutions coupling the lubrication equation with film shape equation and energy equation were obtained to reveal the combined influences of thermal and non-Newtonian character of lubricant on elastohydrodynamic lubrication. Kumar et al. $[16,17]$ dealt with the numerical simulation of thermo-EHL condition in the Rayleigh step bearing. Thermo-EHL involved rheology of the lubricant and deformation of the structure simultaneously under the influences of pressure and temperature. $\mathrm{Xu}$ et al. [18] put forward an analysis method that could determine the best preload at different speeds and combined with the mathematical model of the ball bearing to calculate the appropriate preload. However, the influence of bearing lubrication on the bearing was not considered. In order to improve the bearing stiffness, some researchers [19-22] proposed to use the preload method to improve the bearing stiffness. In recent years, scholars have established the models of the bearing-rub-impact rotor system with time-varying stiffness of rolling bearings and solved the dynamic equation of the system by the Newmark$\beta$ integration method $[23,24]$. At the same time, the angular relationship between the residual vibration displacement and the excitation response displacement was studied, the dynamic bearing stiffness test principle was derived, and the dynamic stiffness of the bearing was tested [25], which provided a new method for measuring the dynamic stiffness of bearings, but the influence of other factors on the stiffness of bearings was not considered.

When the bearing rotates at high speed, the centrifugal force and friction heat are generated, and the lubrication state of the lubricant between rolling elements and rings also changes. However, some above works only calculated the contact stiffness or film stiffness; in addition, only one or two influence factors were considered when calculating stiffness. In this paper, the front bearing of the motorized spindle is taken as the research object. Considering the centrifugal deformation, thermal deformation, and EHL, the modified quasistatic mechanical model of the angular contact ball bearing is put forward. Meanwhile, the film stiffness model considering spin motion is presented. Then, the composite stiffness model combined the contact stiffness and film stiffness in series is established. At last, the influence laws of various factors on the dynamic characteristic parameters of the bearing are studied in more detail, which is necessary theoretical basis for the structure optimal design of the bearing.

\section{Calculation of Bearing Deformation}

The basic structure of the angular contact ball bearing is shown in Figure 1. $\alpha_{o}$ is the outer ring contact angle, $\alpha_{i}$ is the inner ring contact angle, $r_{i}$ is the raceway groove curvature radius of the inner ring, $r_{o}$ is the raceway groove curvature radius of the outer ring, $d_{m}$ is the bearing pitch diameter, and $D_{b}$ is the rolling element diameter.

2.1. Centrifugal Deformation Calculation. When the bearing rotates at high speed, the inner ring and the ball are affected by centrifugal force, which results in radial centrifugal deformation. Meanwhile, the outer ring and the bearing seat become interference fit under the action of temperature rise, and no centrifugal force is generated. Therefore, the radial deformation $\delta_{1}$ of the inner ring due to centrifugal effect is given as follows [26]:

$$
\delta_{1}=\frac{\rho_{i} \omega^{2}}{16 E_{i}}\left[\left(1-v_{i}\right) D_{i}^{2}+\left(3+v_{i}\right) d_{i}^{2}\right] D_{i},
$$

where $\rho_{i}$ is the material density of the inner ring, $E_{i}$ is the elastic modulus of the inner ring, $D_{i}$ is the diameter of the inner ring, $d_{i}$ is the inner diameter of the inner ring, and $v_{i}$ is the Poisson ratio of the inner ring.

2.2. Thermal Deformation Calculation. When the motorized spindle runs at high speed, the power loss of the motor and the friction heat of the bearing can cause the temperature of the shaft and bearing to rise, which leads to the axial and radial thermal deformation of bearing components. In order to facilitate the calculation of thermal deformation, the following assumptions are made:

(1) The outer ring is fixed, and the inner ring rotates. The initial fit between the inner ring and spindle is interference fit, and the initial fit between the outer ring and bearing seat is clearance fit.

(2) Because the width of the inner and outer ring is smaller than the diameter, it is regarded as a thinwalled ring. 


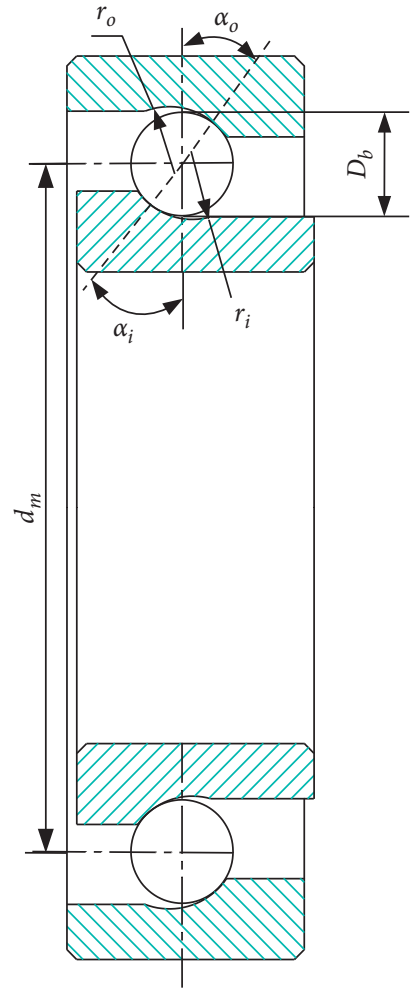

Figure 1: Basic mechanism of angular contact bearing.

(3) The bearing pedestal is simplified as a circular hollow shaft, and the main shaft is calculated as a long cylinder.

Usually, according to Harris' calculation method of thermal deformation of thin-walled ring parts,

$$
u=a d_{T} \Delta T,
$$

where $u$ is the thermal deformation, $a$ is the thermal expansion coefficient, $\Delta T$ is the temperature rise, and $d_{T}$ is the diameter of part.

According to equation (2), the thermal deformation of the inner ring can be obtained as follows:

$$
u_{i}=a_{i} d_{i} \Delta T_{i},
$$

where $u_{i}$ is the thermal deformation of the inner ring, $a_{i}$ is the thermal expansion coefficient of the inner ring, $\Delta T_{i}$ is the temperature rise of the inner ring, and $d_{i}$ is the diameter of the inner ring.

Similarly, the thermal deformation of the outer ring can be written as follows:

$$
u_{o}=a_{o} d_{o} \Delta T_{o},
$$

where $u_{o}$ is the thermal deformation of the outer ring, $a_{o}$ is the thermal expansion coefficient of the outer ring, $\Delta T_{o}$ is the temperature rise of the outer ring, and $d_{o}$ is the diameter of the outer ring.

The thermal deformation of the ball can be described as follows:

$$
u_{b}=a_{b} D_{b} \Delta T_{b}
$$

where $u_{b}$ is the thermal deformation of the ball, $a_{b}$ is the thermal expansion coefficient of the ball, $\Delta T_{b}$ is the temperature rise of the ball, and $D_{b}$ is the diameter of the ball.

From equations (3)-(5), it can be known that when the bearing rotates at high speed, the radial deformation amount $\delta_{2}$ caused by temperature rise is deduced as follows:

$$
\delta_{2}=u_{i}-2 u_{b}-u_{o}
$$

The bearing axial deformation is mainly related to its configuration type, and there are different axial deformations for different configurations. Therefore, the axial deformation $u_{a}$ caused by temperature rise can be expressed as follows:

$$
u_{a}= \pm \frac{a_{h} l_{h} \Delta T_{h}-a_{s} l_{s} \Delta T_{s}}{2},
$$

where $a_{h}$ and $a_{s}$ are the thermal expansion coefficients of bearing housing and the shaft, respectively, $l_{h}$ and $l_{s}$ are the effective contact lengths between the housing hole and the outer ring and between the shaft and the inner ring, respectively, and $\Delta T_{h}$ and $\Delta T_{s}$ are the temperature rise of the housing and the shaft, respectively. Take "+" for dual "O" configuration and "-" for dual " $\mathrm{X}$ " configuration.

According to equations (3)-(7), the temperature rise of each component of the angular contact ball bearing is measured and calculated to obtain the thermal deformation of the inner and outer rings and rolling elements.

2.3. Assembly Deformation Calculation. After the inner ring of the ball bearing and the shaft are assembled by interference fit, in order to ensure the assembly reliability, the stress produced by the assembly interference is zero. Therefore, the radial deformation of the inner ring after bearing assembly is expressed as follows [27]:

$$
\begin{aligned}
\delta_{3} & =\frac{2 r_{1}^{2} r_{2}}{E_{i}\left(r_{2}^{2}-r_{1}^{2}\right)} P, \\
P & =\frac{\delta_{4}}{2 r_{1}}\left[\frac{1}{E_{i}}\left(1-v_{s}\right)+\frac{1}{E_{i}}\left(\frac{r_{1}^{2}+r_{2}^{2}}{r_{1}^{2}-r_{2}^{2}}-v_{s}\right)\right],
\end{aligned}
$$

where $P$ is the assembly stress, $\delta_{4}$ is the initial interference, $v_{s}$ is the Poisson ratio of the shaft, and $r_{1}$ and $r_{2}$ are the inner and outer radius of the inner ring, respectively.

According to the above calculations of the centrifugal deformation, thermal deformation, and assembly deformation, the radial bearing deformation $u_{r}$ and axial deformation $u_{a}$ of the bearing can be obtained. The axial deformation $u_{a}$ is shown by equation (7), and the radial deformation is shown by the following equation:

$$
u_{r}=\delta_{1}+\delta_{2}+\delta_{3} \text {. }
$$




\section{Modified Quasistatic Model of Angular Contact Ball Bearing}

3.1. Basic Equations of EHL. When the angular contact ball bearing is running at high speed, there is spin motion on both inner and outer raceways. Suppose the lubrication contact between the ball and the raceway is equivalent to the contact between the infinite plane and the ellipsoid, and the plane is rotated at the spin angular velocity $\omega_{s}$, and the ellipsoid rolls at the average speed $u_{b}$ of the rolling element and the raceway, as shown in Figure 2 [28].

The entrainment velocity $u_{R}$ and $v_{R}$ in $x$-direction and $y$ direction can be obtained from Figure 2:

$$
\begin{aligned}
& u_{R}(x, y)=\frac{\omega_{s} y}{2}+u_{b} \\
& v_{R}(x, y)=\frac{\omega_{s} x}{2}
\end{aligned}
$$

According to the point contact spin motion model of angular contact ball bearings, Reynolds equation of isothermal point contact EHL is selected [29]:

$$
\frac{\partial}{\partial x}\left(\frac{\partial p}{\partial x} \frac{\rho h^{3}}{\eta}\right)+\frac{\partial}{\partial y}\left(\frac{\partial p}{\partial y} \frac{\rho h^{3}}{\eta}\right)=12 \frac{\partial}{\partial x}\left(u_{R} \rho h\right)+12 \frac{\partial}{\partial y}\left(v_{R} \rho h\right),
$$

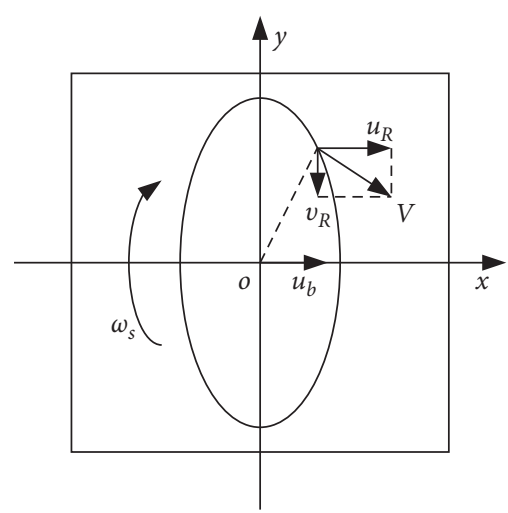

FIGURE 2: Ball speed vector in spinning motion.

where $p$ is the pressure, $\rho$ is the density of lubricant, $h$ is the lubricant film thickness, and $\eta$ is the lubricant viscosity. In addition, the parameters are expressed as follows:

$$
\begin{aligned}
& h(x, y)=h_{0}+\frac{x^{2}}{2 R_{x}}+\frac{y^{2}}{2 R_{y}}+\frac{2}{\pi E} \iint \frac{p(s, t) \mathrm{d} s \mathrm{~d} t}{\sqrt{(x-s)^{2}+(y-t)^{2}}}, \\
& \eta=\eta_{0} \exp \left\{\left(\ln \eta_{0}+9.67\right)\left[\left(1+\frac{p}{p_{0}}\right)^{\left(\alpha_{p} / 5.1 \times 10^{-9}\left(\ln \eta_{0}+9.67\right)\right)}-1\right]\right\} \\
& \rho=\rho_{0}\left(1+\frac{0.6 \times 10^{-9} p}{1+1.7 \times 10^{-9} p}\right) \\
& \int_{x_{0}}^{x_{e}} \int_{y_{0}}^{y_{e}} p \mathrm{~d} x \mathrm{~d} y=w, \\
& E=\left(\frac{1-v_{1}^{2}}{2 E_{1}}+\frac{1-v_{2}^{2}}{2 E_{2}}\right)^{-1} \\
& H_{\text {mins }}=8.9 \times 10^{-3} U^{0.72} G^{0.8001} k^{1.616}\left(1+1.043 e^{-664.016 W}\right) \\
& \left(1+21.459 e^{-0.3 k}\right) \times\left(1-1.3101 \times 10^{10} \Omega_{s}^{1.024}\right), \\
& h_{\text {mins }}=H_{\text {mins }} \times R_{x} \text {, } \\
& \Omega_{s}=\frac{\omega_{s} \eta_{0}}{E} \\
& U=\frac{u_{b} \eta_{0}}{E R_{x}}
\end{aligned}
$$

where $h_{0}$ is the central film thickness between two solid bodies, $R_{x}$ and $R_{y}$ are the equivalent curvature radius of the ball along $x$-direction and $y$-direction, $E$ is the equivalent elastic modulus, $\eta_{0}$ denotes the initial dynamic viscosity, $\alpha_{p}$ is the viscosity-pressure coefficient, $\rho_{0}$ is the ambient density, $E_{1}$ and $E_{2}$ are the elasticity modulus of the ring and ball, and $v_{1}$ and $v_{2}$ are Poisson's ratio of the ring and ball, respectively.

According to Reference [30], the dimensionless minimum film thickness considering spin motion is described as follows: 


$$
\begin{aligned}
& W=\frac{w}{E R_{x}^{2}}, \\
& G=\alpha_{p} E,
\end{aligned}
$$

where $U$ is the velocity parameter, $G$ is the material parameter, $k$ is the ellipticity, $\Omega_{s}$ is the spinning angular velocity parameter, and $H_{\text {mins }}$ is the minimum film thickness when considering spin motion. The values of these parameters are shown in Table 1.

3.2. Basic Equations of Quasistatics. The dynamic model of the angular contact ball bearing is established with the following assumptions:

(1) The center of mass of the ball and the inner and outer ring of the bearing coincide with the geometric center, respectively

(2) The rolling element can spin and rotate

(3) The parameters of lubrication are known

Figure 3 shows the film thickness and pressure distribution of EHL and Hertz contact in the contact zone. In Figure $3, \Delta \delta$ represents the displacement change of the center point of the rolling element under the action of EHL. $\delta$ is the elastic deformation under the Hertz contact state. $h_{0}$ and $h_{\text {min }}$ are the center film thickness and the minimum film thickness, respectively. The center film thickness is shown in equation (19), and the relationship among the displacement change of the ball center point $\Delta \delta$, the elastic deformation $\delta$, and the central film thickness $h_{0}$ is shown in equation (20).

$$
\begin{aligned}
h_{0}= & 1.397 W^{0.678}\left(1-1.418 \times 10^{5} U^{0.677}\right) \\
& \cdot\left[\exp \left(-4.443 \times 10^{-5} G\right) \times 0.155+1\right] k^{-0.364} \\
& \cdot[1-\exp (-2.628 k)] \times\left(1+1.249 \times 10^{19} \Omega_{s}^{2.274}\right) \times R,
\end{aligned}
$$

$$
\Delta \delta=\delta-h_{0} .
$$

In this paper, on the basis of Jones' internal displacement deformation relationship of the angular contact ball bearing, the modified quasistatic model is built by considering the central film thickness under the EHL situation, which makes the quasistatic model more perfect. Figure 4 shows the displacement deformation relationship of the bearing considering the EHL.

$$
\begin{aligned}
L_{i j} & =\left(f_{i}-0.5\right) D_{b}+\delta_{i j}-h_{i j}, \\
L_{o j} & =\left(f_{o}-0.5\right) D_{b}+\delta_{o j}-h_{o j},
\end{aligned}
$$

where $L_{i j}$ and $L_{o j}$ are the distance between the curvature centers of the inner and outer rings and the final position of the ball center when EHL is considered. $\delta_{i j}$ and $\delta_{o j}$ are the contact deformation at the inner and outer ring, respectively. $h_{i j}$ and $h_{o j}$ are the center film thickness of the inner and outer rings, respectively.
TABLE 1: Values of the parameters.

\begin{tabular}{lc}
\hline Parameter & Value \\
\hline$K$ & $1-9.5$ \\
$u_{b}(\mathrm{~m} / \mathrm{s})$ & $1-40$ \\
$w(\mathrm{~N})$ & $200 \sim 10^{4}$ \\
$w_{s}(\mathrm{rad} / \mathrm{s})$ & $0-500$ \\
$G$ & $2500-5000$ \\
$W$ & $3.23 \times 10^{-4} \sim 1.94 \times 10^{-3}$ \\
$U$ & $1.68 \times 10^{-11}-6.74 \times 10^{-10}$ \\
$\Omega_{s}$ & $0-4.41 \times 10^{-11}$ \\
\hline
\end{tabular}

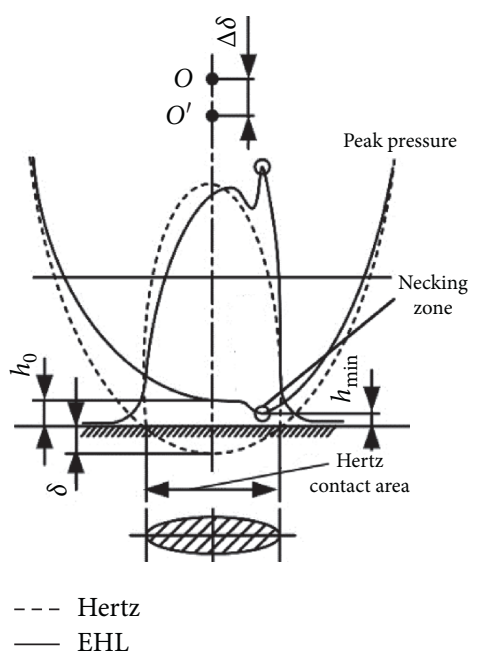

Figure 3: Lubricant film thickness and pressure distribution under EHL and Hertz contact state.

Considering the influences of centrifugal deformation, EHL, and thermal deformation, the distances between the loci of the inner and outer ring groove curvature center are expressed as follows:

$$
\begin{aligned}
& A_{1 j}=B D_{b} \sin \alpha_{0}+\delta_{a}+\theta \Re_{i} \cos \psi_{j}+u_{a}, \\
& A_{2 j}=B D_{b} \cos \alpha_{0}+\delta_{r} \cos \psi_{j}+u_{r},
\end{aligned}
$$

where $A_{1 j}$ and $A_{2 j}$ are the axial and radial distances between the inner and outer ring groove curvature center, respectively, $\alpha_{0}$ is the initial contact angle, $\delta_{a}$ and $\delta_{r}$ are the relative axial and radial displacements of the inner and outer rings, respectively, $\theta$ is the relative angular displacement, and $\Psi_{j}$ is the azimuth of $j$-th rolling element.

According to the Pythagorean theorem, the geometric compatibility equations of the groove contact deformation are obtained as follows [3]:

$$
\begin{aligned}
\left(A_{1 j}-X_{1 j}\right)^{2}+\left(A_{2 j}-X_{2 j}\right)^{2} & =L_{i j}^{2}, \\
X_{1 j}^{2}+X_{2 j}^{2} & =L_{o j}^{2},
\end{aligned}
$$

where $X_{1 j}$ and $X_{2 j}$ are the axial and radial distances between the rolling element center and the outer ring groove curvature center, respectively.

From Figure 5, according to the outer raceway control theory and the quasistatic mechanics, the equilibrium of 


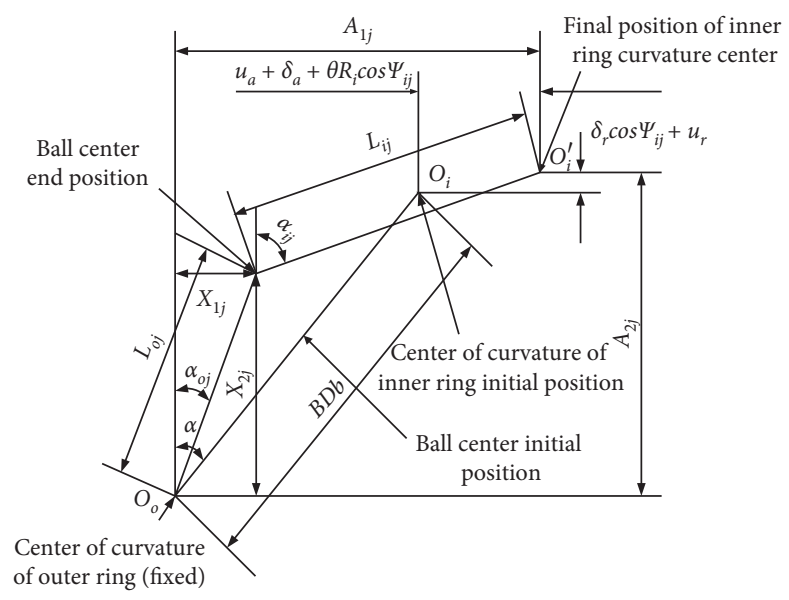

FIGURE 4: Internal displacement change of ball bearings considering EHL.

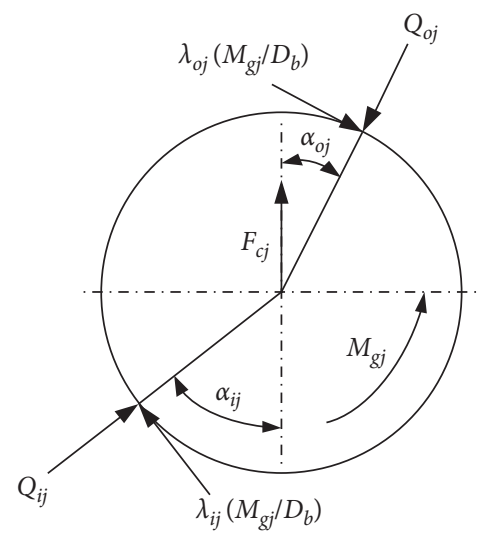

FIgURE 5: Loads on the $j$-th ball rolling body.

forces of the $j$-th ball in the horizontal and vertical directions is given as follows:

$$
\begin{aligned}
Q_{i j} \sin \alpha_{i j}-Q_{o j} \sin \alpha_{o j}-\frac{M_{g j}}{D_{b}}\left(\lambda_{i j} \cos \alpha_{i j}-\lambda_{o j} \cos \alpha_{o j}\right) & =0 \\
Q_{o j} \cos \alpha_{i j}-Q_{o j} \cos \alpha_{o j}-\frac{M_{g j}}{D_{b}}\left(\lambda_{i j} \sin \alpha_{i j}-\lambda_{o j} \sin \alpha_{o j}\right) & =-F_{c j}, \\
Q_{i j} & =K_{i j} \delta_{i j}^{1.5}, \\
Q_{o j} & =K_{o j} \delta_{o j}^{1.5}, \\
F_{c j} & =\frac{1}{2} m_{b} d_{m} \omega^{2}\left(\frac{\omega_{m}}{\omega}\right)_{j}^{2}, \\
M_{g j} & =J\left(\frac{\omega_{r}}{\omega}\right)_{j}\left(\frac{\omega_{m}}{\omega}\right)_{j} \omega^{2} \sin \beta,
\end{aligned}
$$


where $Q_{i j}$ and $Q_{o j}$ are the contact loads of the inner and outer rings, respectively, and $K_{i j}$ and $K_{o j}$ are the contact deformation coefficients of the inner and outer rings, respectively.
According to the above analysis of the relationship between the force and deformation of the ball, the force balance equations of the whole bearing are obtained as follows:

$$
\begin{aligned}
F_{a} & =\sum_{j=1}^{j=Z}\left(Q_{i j} \sin \alpha_{i j}-\frac{\lambda_{i j} M_{g j}}{D_{b}} \cos \alpha_{i j}\right), \\
F_{r} & =\sum_{j=1}^{j=Z}\left(Q_{i j} \sin \alpha_{i j}-\frac{\lambda_{i j} M_{g j}}{D_{b}} \cos \alpha_{i j}\right) \cos \psi_{i j}, \\
M & =\sum_{j=1}^{j=Z}\left[\left(Q_{i j} \sin \alpha_{i j}-\frac{\lambda_{i j} M_{g j}}{D_{b}} \cos \alpha_{i j}\right) \Re_{i}+\frac{\lambda_{i j} M_{g j}}{D_{b}} r_{i}\right] \cos \psi_{i j},
\end{aligned}
$$

where $Z$ is the number of balls, $F_{a}$ is the axial load of the bearing, $F_{r}$ is the radial load of the bearing, $M_{g j}$ is the gyroscopic moment of the bearing, and $M$ is the moment of the bearing.

In order to analyze the dynamic characteristics of the angular contact ball bearing under the condition of position preloading, the displacement $\delta_{a}^{\prime}$ of the rolling element relative to the raceway should be calculated according to equations (33) and (34). Then, according to the bearing internal load-deformation and nonlinear equations (30)-(32), the Newton-Raphson method is adopted to solve the bearing characteristic parameters. Figure 6 is the calculation flow chart. Equation (35) is the iterative formula of the contact angle.

$$
\begin{gathered}
\delta_{a}^{\prime}=\frac{B D_{b} \sin \left(\alpha-\alpha^{\prime}\right)}{\cos \alpha}, \\
\frac{F_{a}}{Z K_{n} D_{b}^{2}}=\sin \alpha\left(\frac{\cos \alpha^{\prime}}{\cos \alpha}-1\right)^{1.5}, \\
\alpha^{(k+1)}=\alpha^{(k)}+\frac{\left(F_{a} /\left(Z K_{n}\left(B D_{b}\right)^{1.5}\right)\right)-\sin \alpha\left(\left(\cos \alpha^{\prime} / \cos \alpha\right)-1\right)^{1.5}}{\cos \alpha\left(\left(\cos \alpha^{\prime} / \cos \alpha\right)-1\right)^{1.5}+1.5 \tan ^{2} \alpha\left(\left(\cos \alpha^{\prime} / \cos \alpha\right)-1\right)^{0.5} \cos \alpha^{\prime}}
\end{gathered}
$$

where $\alpha$ is the contact angle after preloading, $\alpha^{\prime}$ is the contact angle before preloading, $K_{n}$ is the equivalent load-deformation coefficient, $\alpha^{(k+1)}$ is the new contact angle after iteration, and $\alpha^{(k)}$ is the contact angle before iteration.

According to the calculation process in Figure 6, the contact angle, contact load, and deformation of the inner and outer raceways of angular contact ball bearings can be obtained. When the Newton-Raphson iterative method is used to calculate the dynamic parameters of bearings, the initial values of the dynamic parameters should be given first. The initial value of each parameter is shown in Table 2.

\section{Stiffness Model of Angular Contact Ball Bearing}

4.1. Film Stiffness Model. According to the definition of stiffness, the basic formula is as follows [28]:

$$
K_{\text {film }}=\lim _{\delta \longrightarrow 0} \frac{\Delta w}{\delta}=-\frac{\mathrm{d} w}{\mathrm{~d} h_{\min }} .
$$

From equations (13)-(18), the minimum film thickness of the angular contact ball bearing can be obtained under the spinning condition, and then the calculation formula of the film stiffness can be derived as follows:

$$
K_{\text {film } s}=-\frac{\mathrm{d} w_{\text {film } s}}{\mathrm{~d} h_{\min s}}=\frac{0.16219 E R_{x}^{3}}{\left[107.7 R_{x} h_{\min s}-0.9588 G^{0.8001} \times U^{0.72} k^{1.616}\left(1+21.459 e^{-0.3 k}\right) \times\left(1-1.3101 \times 10^{10} \Omega_{s}^{1.024}\right)\right]} .
$$




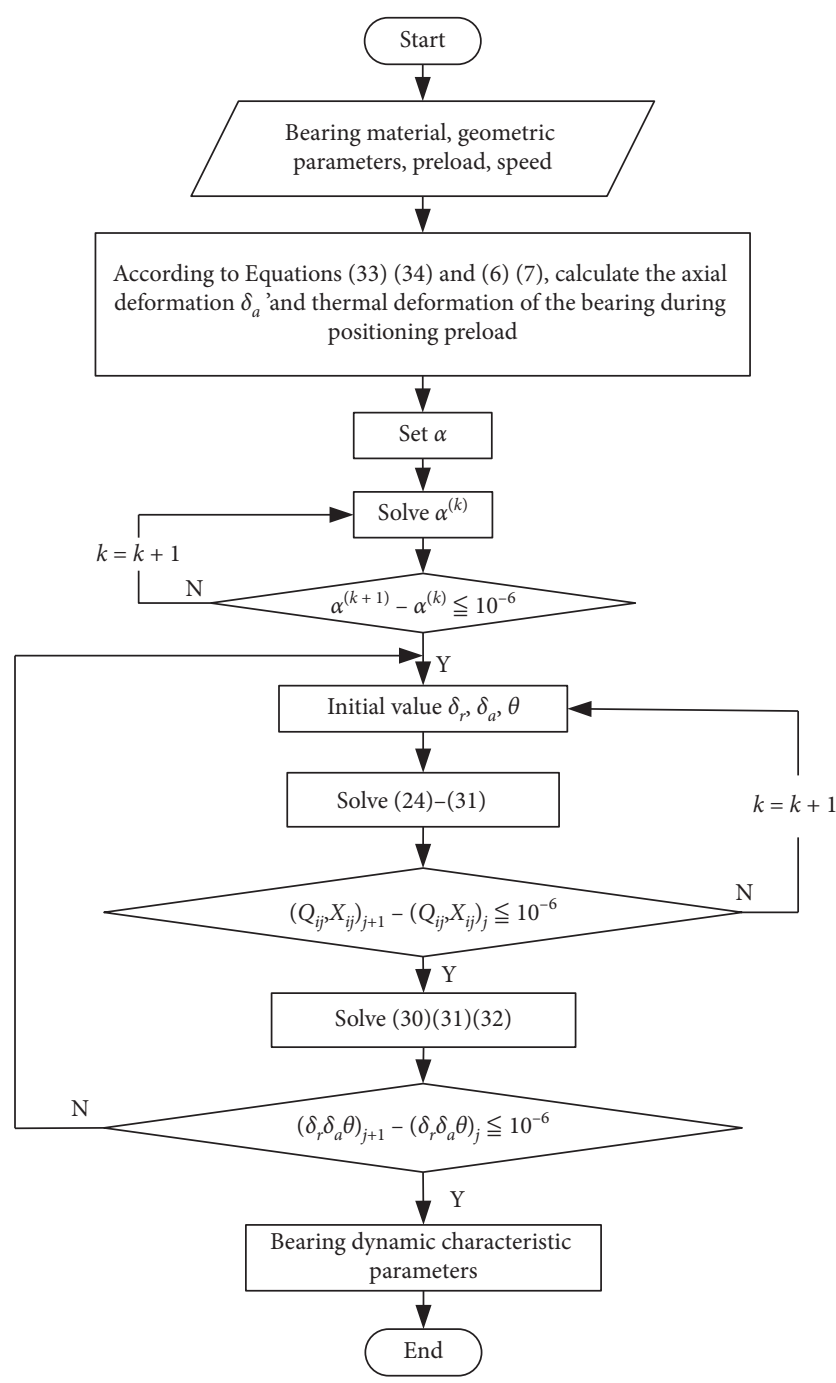

FIGURE 6: Calculation flowchart.

TABLE 2: Initial values of the parameters.

\begin{tabular}{lccccc}
\hline Parameter & $\alpha_{i j}\left({ }^{\circ}\right)$ & $\alpha_{o j}\left({ }^{\circ}\right)$ & $\delta_{a}(\mathrm{~m})$ & $\delta_{r}(\mathrm{~m})$ & $\theta(\mathrm{m})$ \\
\hline Value & 15 & 15 & $10^{-6}$ & $10^{-6}$ & $10^{-6}$ \\
\hline
\end{tabular}

4.2. Contact Stiffness Model. Under normal working conditions, the load on the bearing is nonlinear with its corresponding deformation. According to the basic definition of bearing stiffness, it can be expressed as the differential of load to its displacement in corresponding direction. According to the calculation results in Section 3.2, the contact load and deformation can be obtained, and then the contact stiffness of the inner and outer rings can be calculated, which are expressed by equations (38) and (39). The contact stiffness of the whole bearing is formed by the contact stiffness of the inner and outer rings in series, which is obtained by equation (40).

$$
\begin{gathered}
K_{c i j}=1.5 \Gamma_{i j}^{-1}\left[\frac{9}{\sum \rho_{i j} \Sigma_{i j}}\left(\frac{1}{\pi \kappa_{i j} E}\right)^{2}\right]^{-(1 / 3)} Q_{i j}^{1 / 3}, \\
K_{c o j}=1.5 \Gamma_{o j}^{-1}\left[\frac{9}{\sum \rho_{o j} \Sigma_{o j}}\left(\frac{1}{\pi \kappa_{o j} E}\right)^{2}\right]^{-(1 / 3)} Q_{o j}^{1 / 3}, \\
K_{c}=\frac{K_{c i j} K_{c o j}}{K_{c i j}+K_{c o j}} .
\end{gathered}
$$

4.3. Composite Stiffness Model. The stability of the highspeed angular contact ball bearing is directly affected by the speed of the bearing and the EHL. In order to establish the bearing stiffness model which is closer to the actual working condition, the film stiffness and contact stiffness are connected in series to build the bearing composite stiffness model. Because of the preload and high speed, the stiffness between the rolling element and the inner ring is different from that between the rolling element and the outer ring. Therefore, when calculating the composite stiffness, it is necessary to calculate the contact stiffness and film stiffness of the inner and outer rings, respectively.

The stiffness $K_{i j}$ composed of the film stiffness and contact stiffness between the ball and the inner ring is expressed as follows:

$$
K_{i j}=\frac{\left(K_{c}\right)_{i j}\left(K_{\mathrm{film} s}\right)_{i j}}{\left(K_{c}\right)_{i j}+\left(K_{\mathrm{film} s}\right)_{i j}}
$$

where $\left(K_{c}\right)_{i j}$ and $\left(K_{\text {films }}\right)_{i j}$ are the contact stiffness and film stiffness with spinning motion between the $j$-th roller and the inner ring, respectively.

Similarly, the stiffness $K_{o j}$ composed of the film stiffness and contact stiffness between the ball and the outer ring is described as follows:

$$
K_{o j}=\frac{\left(K_{c}\right)_{o j}\left(K_{\mathrm{film} s}\right)_{o j}}{\left(K_{c}\right)_{o j}+\left(K_{\mathrm{film} s}\right)_{o j}}
$$

where $\left(K_{c}\right)_{o j}$ and $\left(K_{\text {films }}\right)_{o j}$ are the contact stiffness and film stiffness with spinning motion between the $j$-th roller and the outer ring, respectively.

According to the stiffness of the inner ring and outer ring obtained from (41) and (42), the radial and axial stiffness of the inner and outer rings can be obtained as follows:

$$
\begin{gathered}
\left\{\begin{array}{l}
K_{r i j}=K_{i j} \cos ^{2} \alpha_{i j}, \\
K_{a i j}=K_{i j} \sin ^{2} \alpha_{i j},
\end{array}\right. \\
\left\{\begin{array}{l}
K_{r o j}=K_{o j} \cos ^{2} \alpha_{o j}, \\
K_{a o j}=K_{o j} \sin ^{2} \alpha_{o j} .
\end{array}\right.
\end{gathered}
$$

The combined radial, axial, and angular stiffness of ball bearings can be derived from equations (43) and (44). 


$$
\begin{aligned}
K_{r} & =\sum_{j=1}^{j=Z} \frac{K_{r i j} K_{r o j}}{K_{r i j}+K_{r o j}} \cos ^{2}\left[\frac{2 \pi}{Z}(j-1)\right], \\
K_{a} & =\sum_{j=1}^{j=Z} \frac{K_{a i j} K_{a o j}}{K_{a i j}+K_{a o j}}, \\
K_{\theta} & =\frac{d_{m}^{2}}{4} \sum_{j=1}^{j=Z} \frac{K_{a i j} K_{a o j}}{K_{a i j}+K_{a o j}} \cos ^{2}\left[\frac{2 \pi}{Z}(j-1)\right],
\end{aligned}
$$

where $K_{r}, K_{a}$, and $K_{\theta}$ are the radial, axial, and angular composite stiffness of the ball bearing, respectively.

\section{Results and Discussion}

5.1. Validation of the Present Model. In order to verify the accuracy of the proposed bearing model, the 7012/CD type bearing in Reference [31] is adopted. According to the calculation process shown in Figure 6 and the stiffness model of angular contact ball bearings, the contact stiffness of the $7012 / \mathrm{CD}$ bearing is calculated under the preload of $100 \mathrm{~N}$, and the results are compared with the results in Reference [31], which is shown in Figure 7. From Figure 7, it can be seen that the calculation results are consistent with that of the reference, the maximum error is about $1.67 \%$, and the correctness of the model and calculation process in this paper are verified.

According to the above dynamic characteristic parameter equations and stiffness model, influences of preload, EHL, and deformations on the dynamic characteristics and stiffness of the angular contact ball bearing under the condition of positioning preload are investigated. The angular contact ball bearing H7013C is the research object, and the bearing structure and material parameters are shown in Table 3.

Members of the research group have measured the temperature rise of each part of the bearing at $15000 \mathrm{r} / \mathrm{min}$ and obtained the thermal deformation of each part of the bearing by ANSYS [32]. On this basis, the temperature rise of each part of the bearing at $24000 \mathrm{r} / \mathrm{min}$ was calculated by the model, and then the radial and axial thermal deformation of the bearing was obtained.

The temperature rise of each part is shown in Figure 8. From Figure 8, the temperature rise of each part is gradually increasing with the increase in speed. But, at the same speed, the temperature rise of the inner ring is higher than that of the roller and outer ring. This is because the inner ring is directly installed with the shaft using the interference fit, and the temperature of the shaft is directly transmitted to the inner ring. The rolling element moves along the inner and outer raceways, which makes its temperature rise higher. The temperature rise of the outer ring mainly comes from the heat transmission of the rolling element, and part of the heat is taken away by the cooling system. As a result, the temperature of the outer ring is lower than that of the inner ring and rolling element.

Figure 9 shows the axial and radial thermal deformation of the ball bearing. It can be seen that the axial and radial

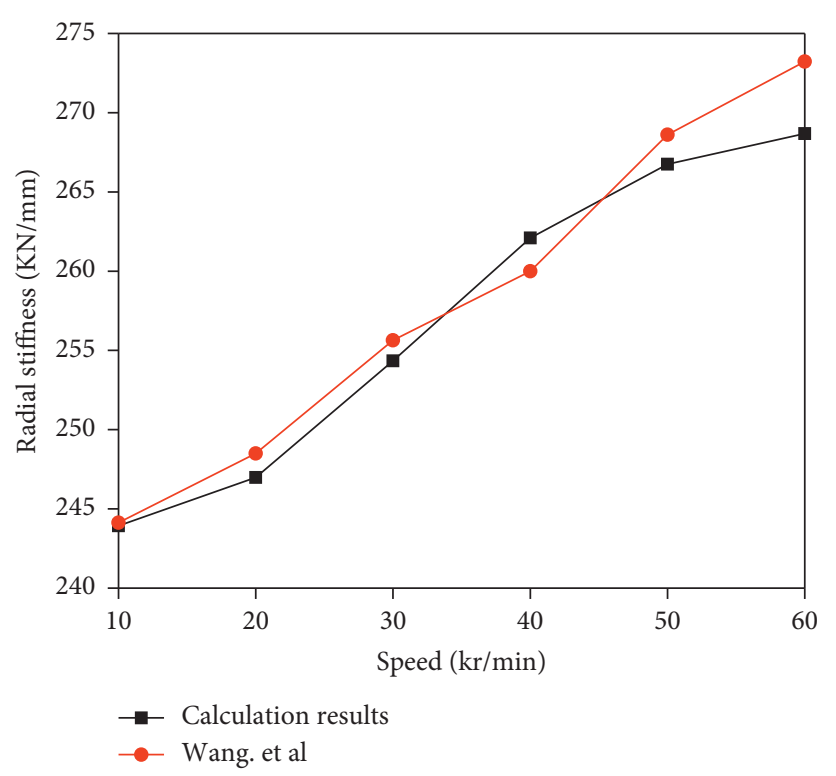

Figure 7: Comparison of two different models.

TABLE 3: Basic parameters of angular contact ball bearings H7013C.

\begin{tabular}{lc}
\hline Parameter & Value \\
\hline Inner ring diameter $(\mathrm{mm})$ & 65 \\
Outer ring diameter $(\mathrm{mm})$ & 100 \\
Ball diameter $(\mathrm{mm})$ & 8.73 \\
Number of balls & 25 \\
Bearing width $(\mathrm{mm})$ & 18 \\
Initial contact angle $\left(^{\circ}\right)$ & 15 \\
Ball density $\left(\mathrm{g} \cdot \mathrm{cm}^{-3}\right)$ & 3.2 \\
Inner ring curvature coefficient & 0.56 \\
Outer ring curvature coefficient & 0.5 \\
\hline
\end{tabular}

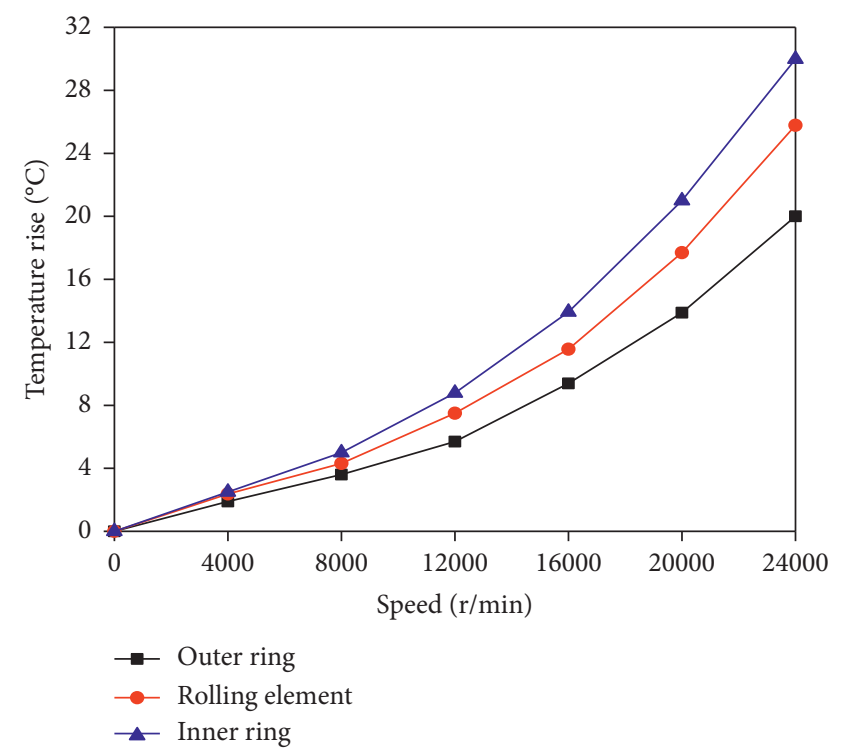

FIgURE 8: Influence of speed on temperature rise of bearing components. 


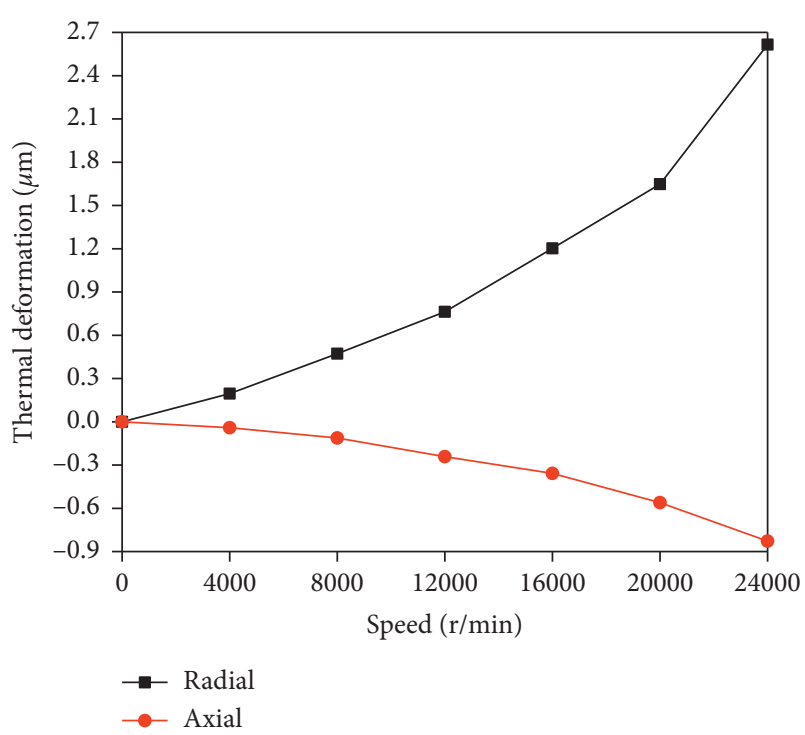

Figure 9: Influence of speed on thermal deformation.

thermal deformation increase with the increase in speed. As the speed increases, the heat production of outer ring, rolling element, and inner ring increases, the temperature increases gradually and then the axial and radial thermal deformation also increase, in which the axial deformation is negative, indicating the separation degree of the rolling body and the raceway.

\subsection{Influences of Multiple Factors on the Dynamic Characteristic Parameters of Bearing}

5.2.1. Influence of Preload on Dynamic Characteristic Parameters of Bearing. When the preload is $400 \mathrm{~N}, 500 \mathrm{~N}$, and $600 \mathrm{~N}$, respectively, the changes of contact angle and contact load of the inner and outer rings are shown in Figures 10 and 11, respectively. In Figure 10, with the increase in speed, the inner contact angle increases and the outer contact angle decreases. This is because as the rotating speed increases, the centrifugal force on the rolling element increases, which makes the rolling element "press" towards the outer ring and "away from" the inner ring. Moreover, the change range of the inner contact angle decreases, while that of the outer contact angle increases. In Figure 11, the contact load of the outer ring increases gradually with the increase in speed, while that of the inner ring decreases. The reason for this phenomenon is that the outer contact angle of the bearing decreases with the increase in speed, which makes the load of the axial preload acting on the outer ring increase, and the load of the inner ring decreases. At the same time, it can be seen that with the increase in axial preload, the contact loads of the inner and outer rings increase.

5.2.2. Influence of EHL on Dynamic Characteristic Parameters of Bearing. When the axial preload is $600 \mathrm{~N}$, the influences of EHL on the contact angle and contact load of the ball bearing with the increase in speed are shown in Figures 12 and 13, respectively. In Figure 12, when EHL is

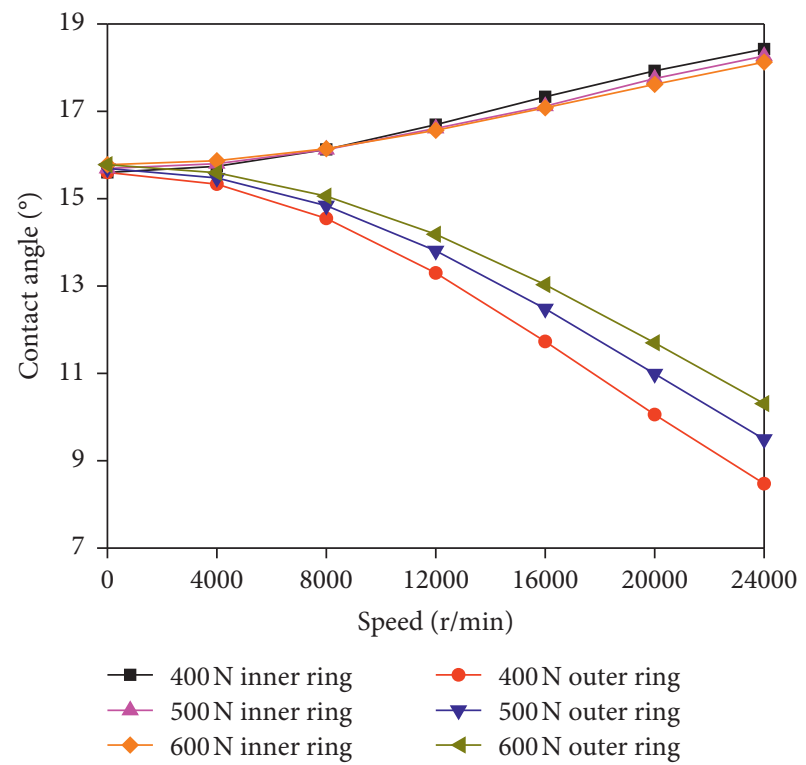

FIGURE 10: Influences of the speed and preload on contact angle.

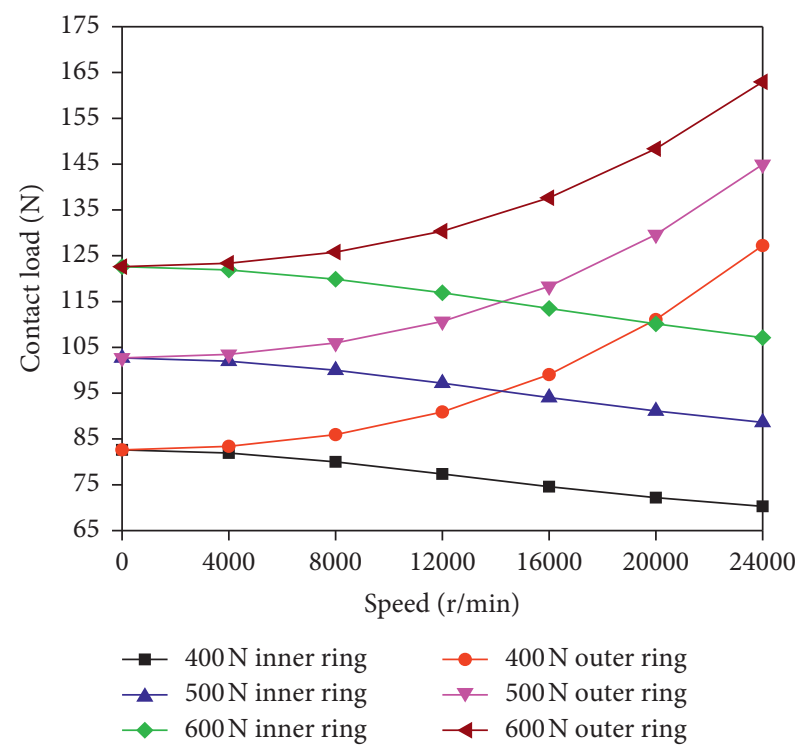

FIGURE 11: Influences of the speed and preload on contact load.

considered, the contact angles of the inner and outer rings are smaller than those without lubrication. When the speed is $24000 \mathrm{r} / \mathrm{min}$, the inner contact angle without lubrication is $18.1264^{\circ}$; while EHL is taken into account, the inner contact angle is $17.2518^{\circ}$, reducing by $4.836 \%$. Similarly, the outer contact angle decreases by $2.798 \%$. This is because considering the effect of EHL, there is lubricating oil in the ball bearing, so that the contact deformation between the inner and outer ring and rolling element is reduced. From Figure 13, considering the effect of EHL, the contact loads of the inner and outer rings are bigger than that without lubrication. This phenomenon is due to the oil film produced by the lubrication between the inner and outer ring and the rolling element, which increases the contact load. 


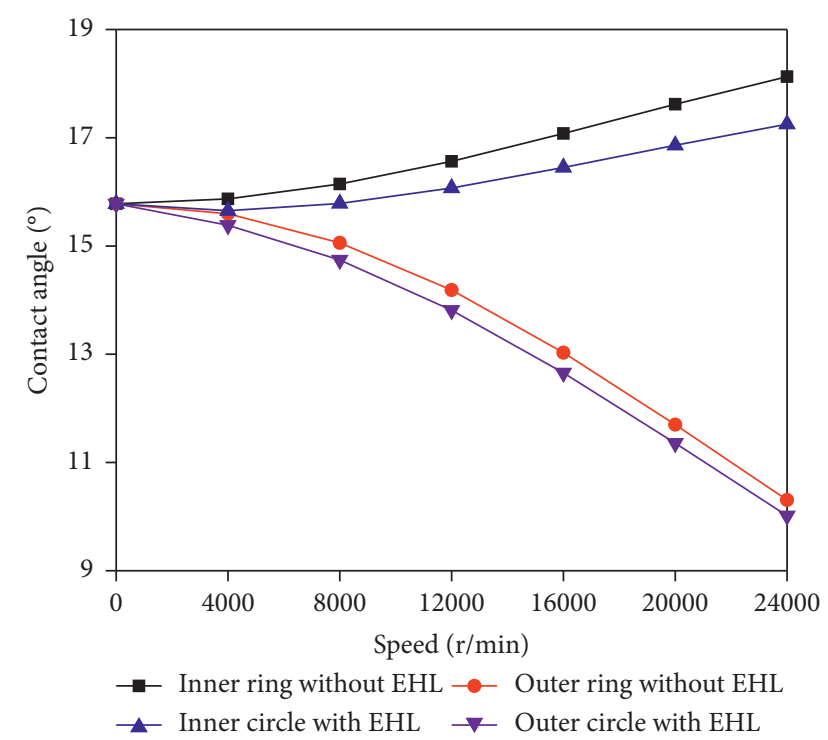

Figure 12: Influences of the speed and EHL on contact angle.

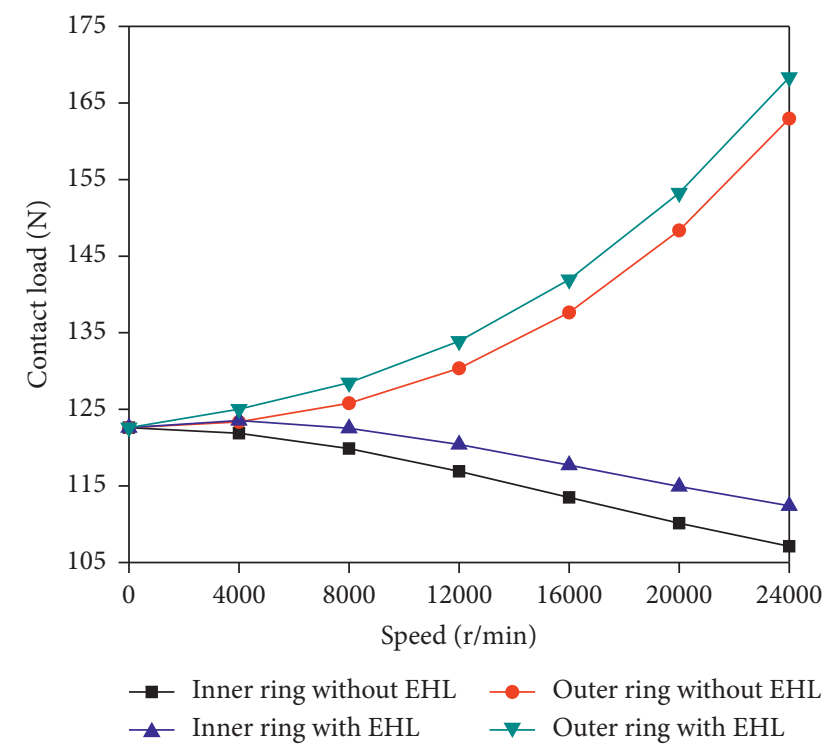

FIGURE 13: Influences of the speed and EHL on contact load.

\subsubsection{Influence of Deformation on Dynamic Characteristic} Parameters of Bearing. According to the above calculation model, the assembly deformation of the ball bearing is too small to be ignored in this section. Figures 14 and 15 show the influences of thermal deformation, centrifugal deformation, and total deformation on the contact angle and contact load when the axial force is 600 N. From Figure 14, with the increase in speed, the contact angle of the inner ring increases and that of the outer ring decreases. Compared with that without deformation, the contact angles of the inner and outer rings considering the deformations reduce. Moreover, when the speed is less than $8000 \mathrm{r} / \mathrm{min}$, the contact angle curves of different working conditions are successively reduced, that is, no deformation, centrifugal deformation, thermal deformation, and total deformation; however, the speed is greater than $8000 \mathrm{r} / \mathrm{min}$, and the contact angle curves are successively reduced, that is, no deformation, thermal deformation, centrifugal deformation, and total deformation. The reason for the phenomenon is that the centrifugal deformation is greater than the thermal deformation within $8000 \mathrm{r} / \mathrm{min}$, but when the bearing speed exceeds $8000 \mathrm{r} / \mathrm{min}$, the thermal deformation is greater than the centrifugal deformation.

From Figure 15, with the increase in speed, the contact load of the inner ring decreases and that of the outer ring increases. Compared with that without deformation, the contact loads of the inner and outer rings considering the deformation are higher. In addition, when the rotating speed is less than $8000 \mathrm{r} / \mathrm{min}$, the contact loads of the inner and outer rings increase successively, that is, no deformation, centrifugal deformation, thermal deformation, and total deformation, but when the speed is greater than $8000 \mathrm{r} / \mathrm{min}$, the contact loads of the inner and outer rings successively increase, that is, no deformation, thermal deformation, centrifugal deformation, and total deformation. The reason is the same as the above explained, and it does not repeat.

5.3. Effect of Spinning Motion on Film Stiffness of Bearing. The spin motion has a great influence on the EHL state of the ball bearing and the film stiffness. Figure 16 shows the effect of spin motion on the film stiffness of the inner and outer rings. The spin angular velocity $\omega_{s}=50 \mathrm{r} / \mathrm{s}$, and the axial force is 600 N. From Figure 16, the film stiffness of the inner and outer rings decreases with the increase in speed, but the film stiffness of the inner and outer rings increases with the consideration of the spin motion. The reason for this phenomenon is that the film thickness of the inner and outer rings decreases due to the spin motion, which leads to the increase in film stiffness. In addition, the contact equivalent curvature radius between the roller and the outer ring is larger, which results in the larger film stiffness of the outer ring than that of the inner ring. This conclusion is consistent with the result of Reference [20], which verifies the correctness of the film stiffness model.

\subsection{Influences of Multiple Factors on Contact Stiffness}

5.4.1. Influence of Preload on Contact Stiffness. Figure 17 illustrates the influence of different axial preloads on the contact stiffness; it can be seen that with the increase in preload, the contact stiffness increases. Due to the increase in the axial preload, the load between the rolling element and the inner and outer raceways increases, and according to the definition of stiffness, the contact stiffness of the bearing increases. Meanwhile, with the increase in preload, the radial deformation is smaller than the axial and angular deformation, which makes the radial stiffness increase and the axial and angular stiffness decrease.

5.4.2. Influence of EHL on Contact Stiffness. EHL plays an important role in reducing friction of ball bearings. Figure 18 shows the influence of EHL on the contact stiffness when the 


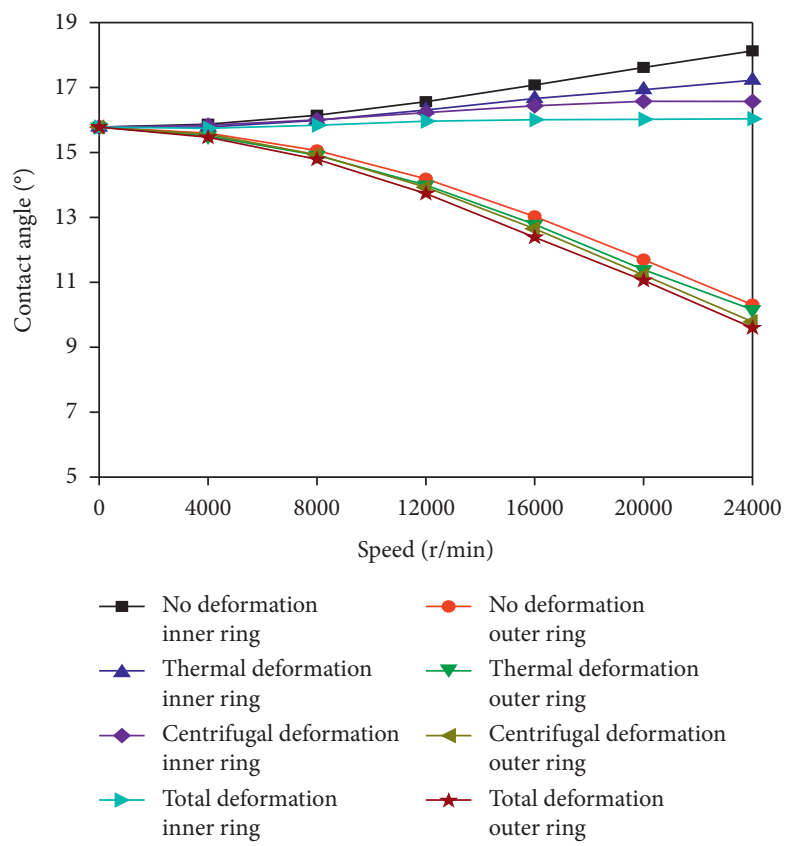

FIGURE 14: Influences of the speed and deformation on the contact angle.

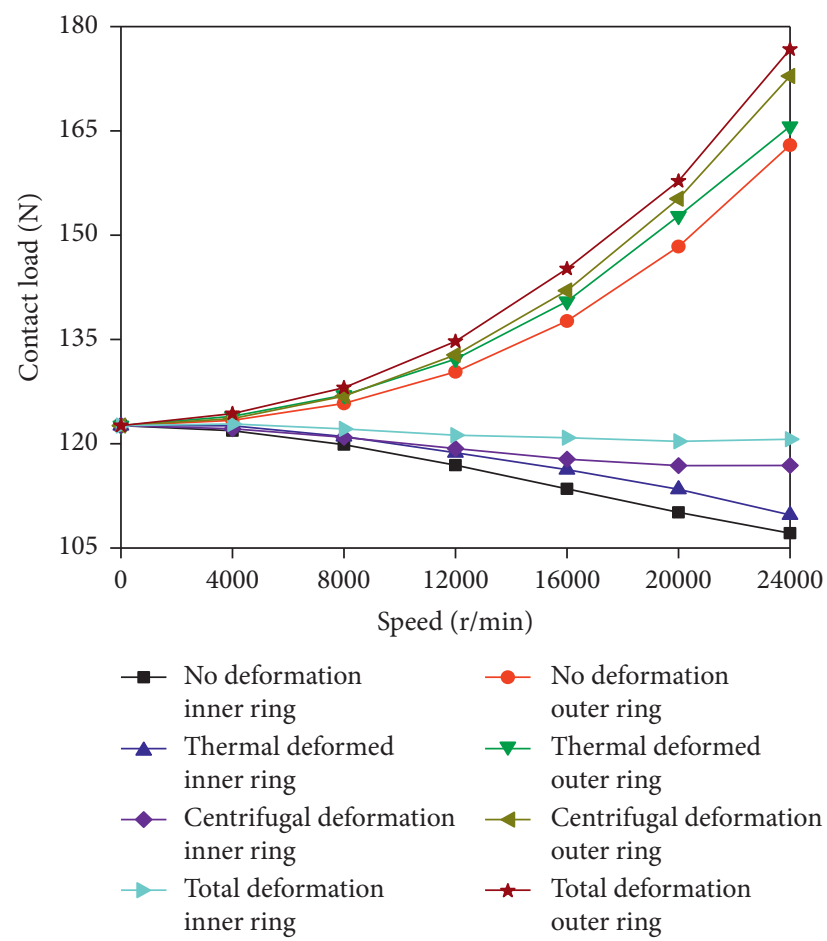

Figure 15: Influences of the speed and deformation on contact load.

axial force is $600 \mathrm{~N}$. In Figure 18(a), the radial stiffness increases with the increase in speed; moreover, the radial stiffness considering lubrication is larger than that without consideration of lubrication. This is mainly due to the reduction of the radial deformation of the inner and outer rings under the condition of positioning preload and EHL.
Figures 18(b) and 18(c) show that compared without consideration of lubrication, the axial and angular stiffness decrease. At the same time, it can be seen that the axial and angular stiffness decrease with the increase in speed. This is because the axial displacement between the center point of the rolling element and the center point of the outer ring 


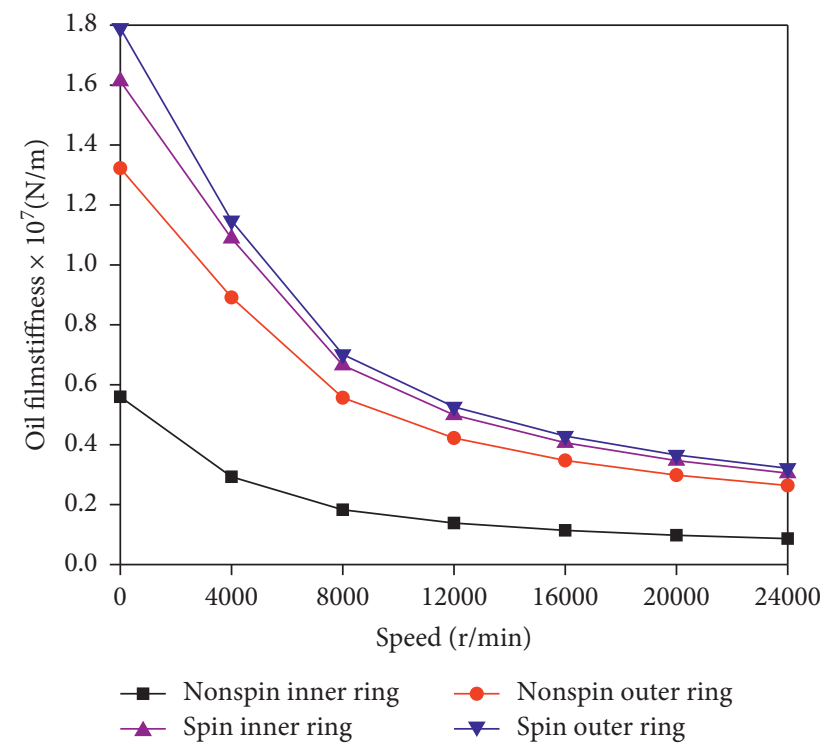

Figure 16: Effects of the speed and spin on film stiffness.
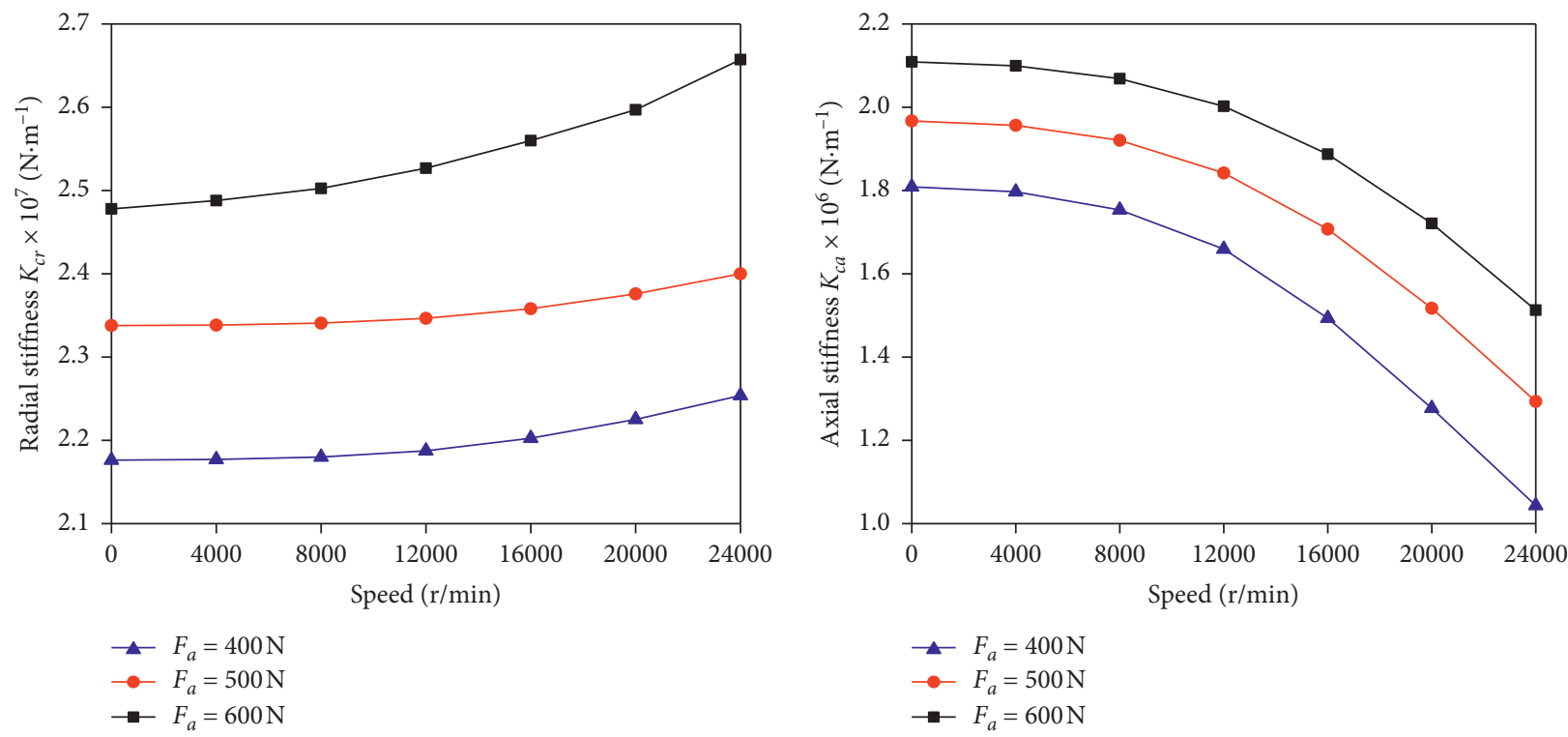

(a)

(b)

FIGURE 17: Continued. 


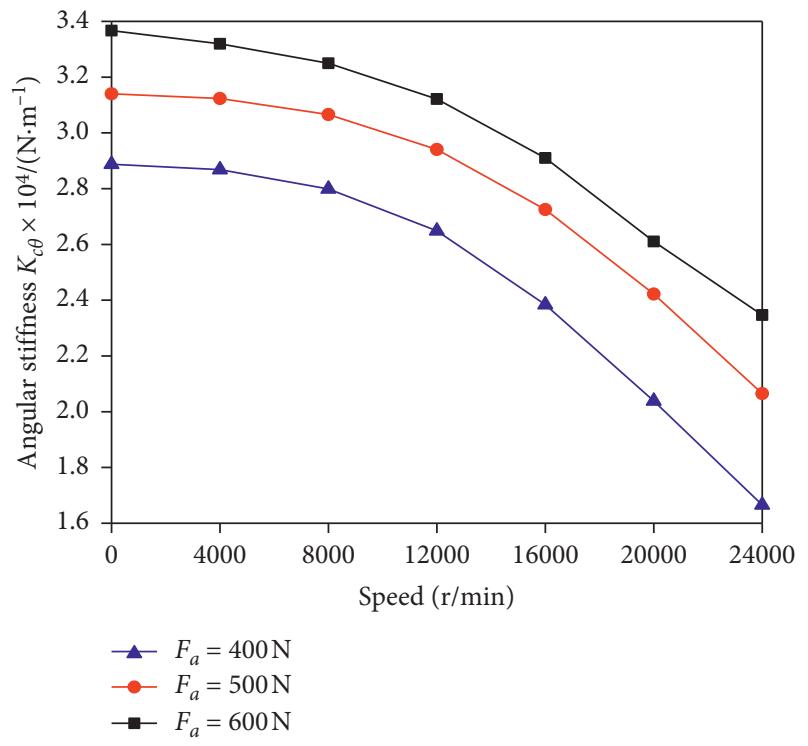

(c)

Figure 17: Influences of the speed and preload on contact stiffness. (a) Radial stiffness. (b) Axial stiffness. (c) Angular stiffness.
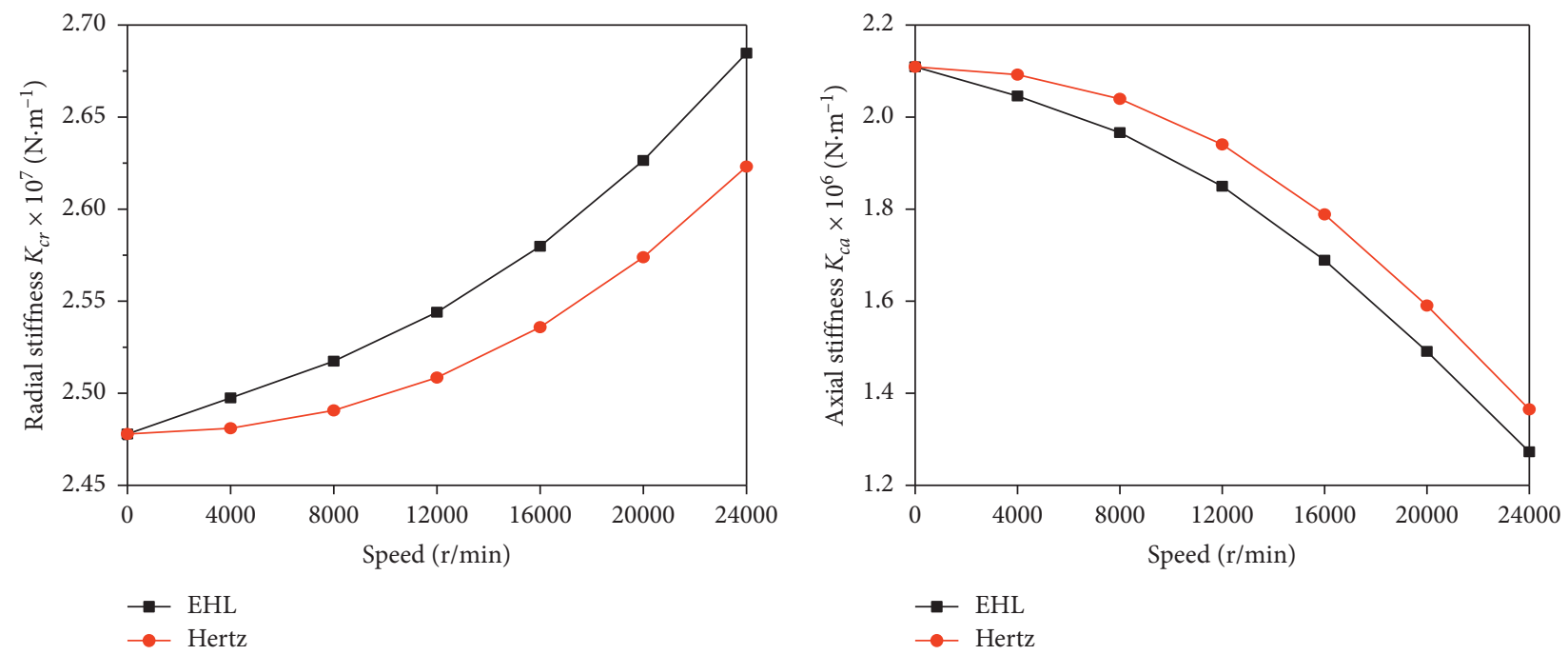

(a)

(b)

FIgURE 18: Continued. 


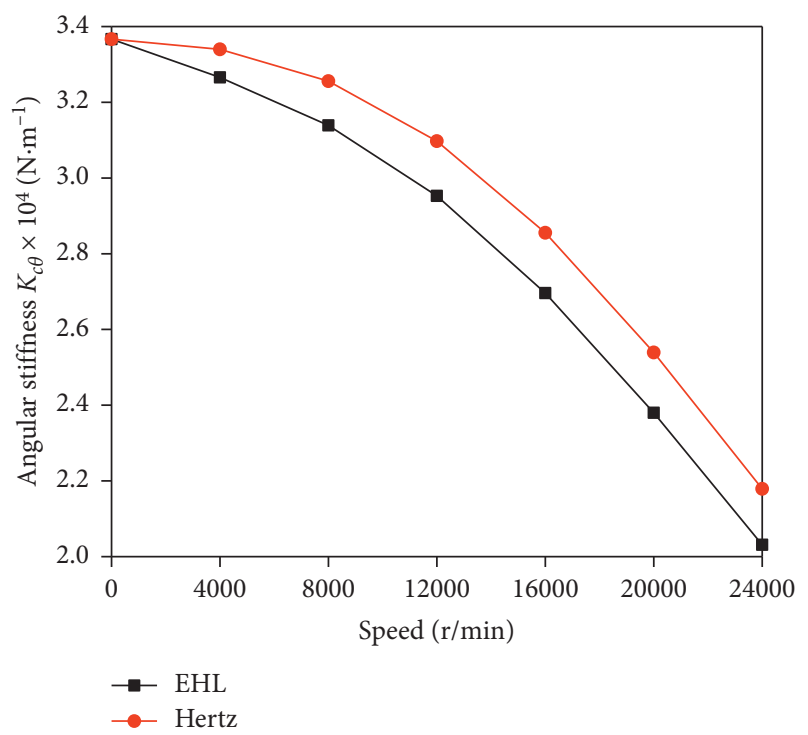

(c)

Figure 18: Influences of the speed and EHL on contact stiffness. (a) Radial stiffness. (b) Axial stiffness. (c) Angular stiffness.

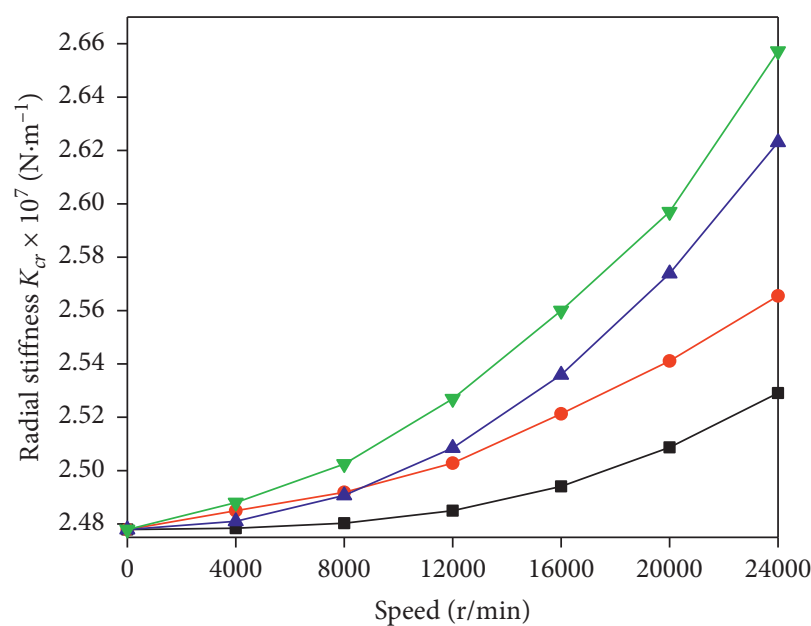

- No deformation

$\leftarrow$ - Centrifugal deformation

- Thermal deformation

$\rightarrow$ Total deformation

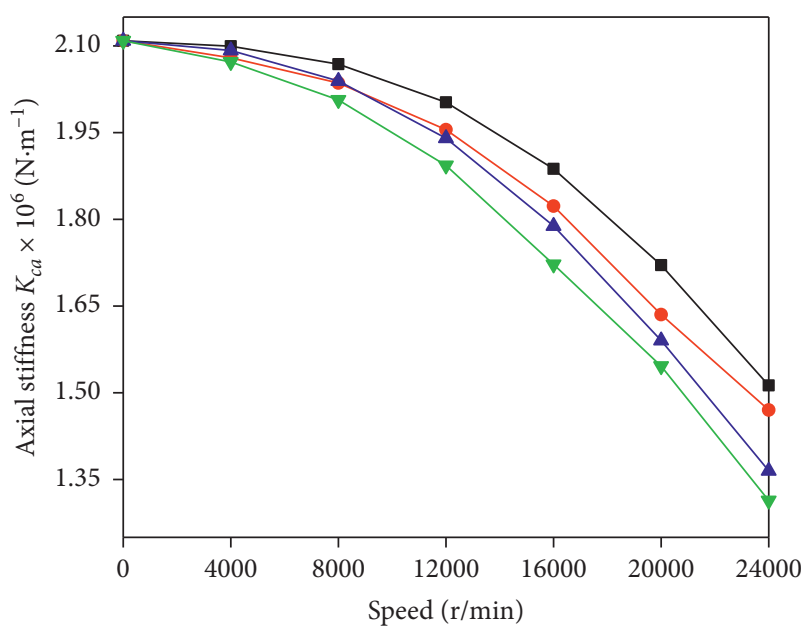

- No deformation

- Thermal deformation

(a)
- Centrifugal deformation

(b)

Figure 19: Continued. 


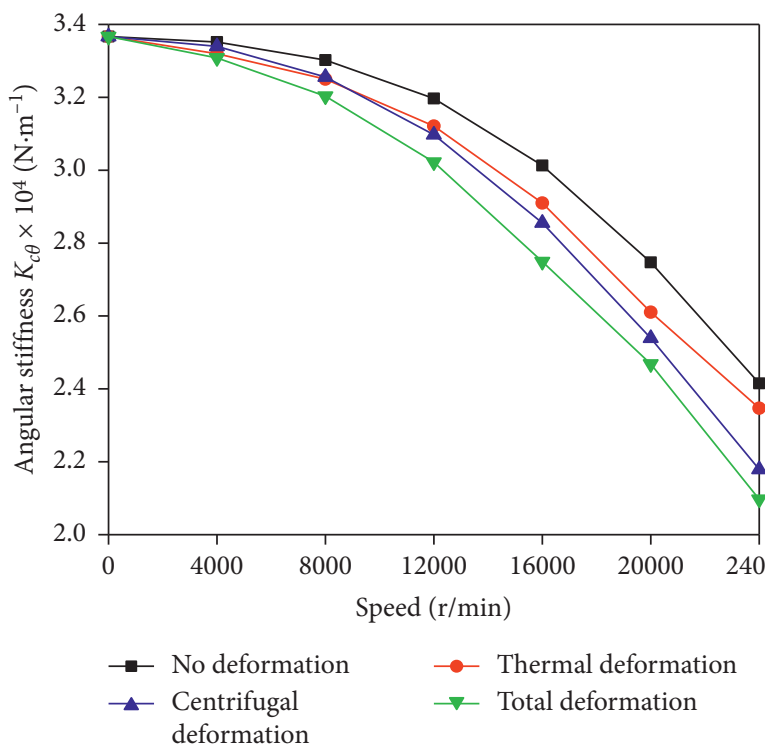

(c)

Figure 19: Influences of the speed and deformation on contact stiffness. (a) Radial stiffness. (b) Axial stiffness. (c) Angular stiffness.

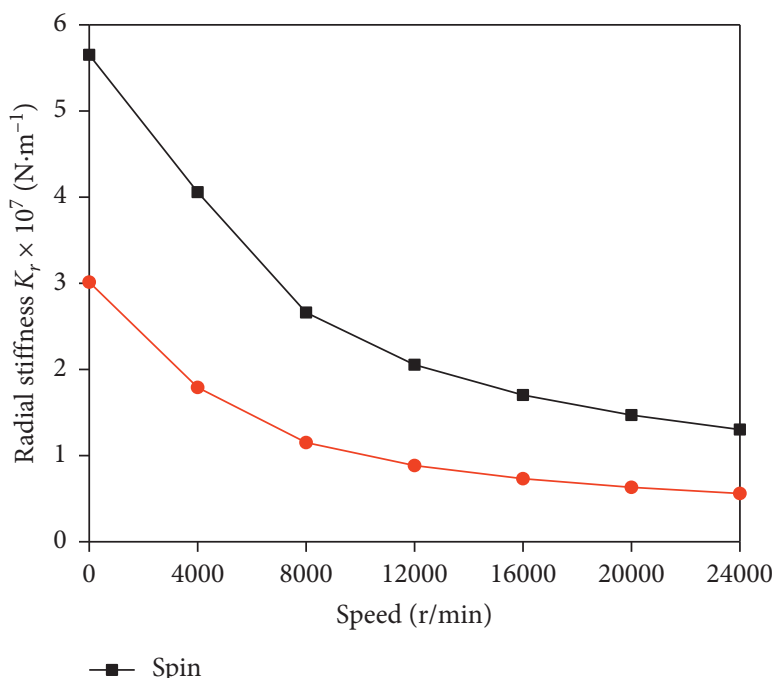

$\rightarrow$ No-spin

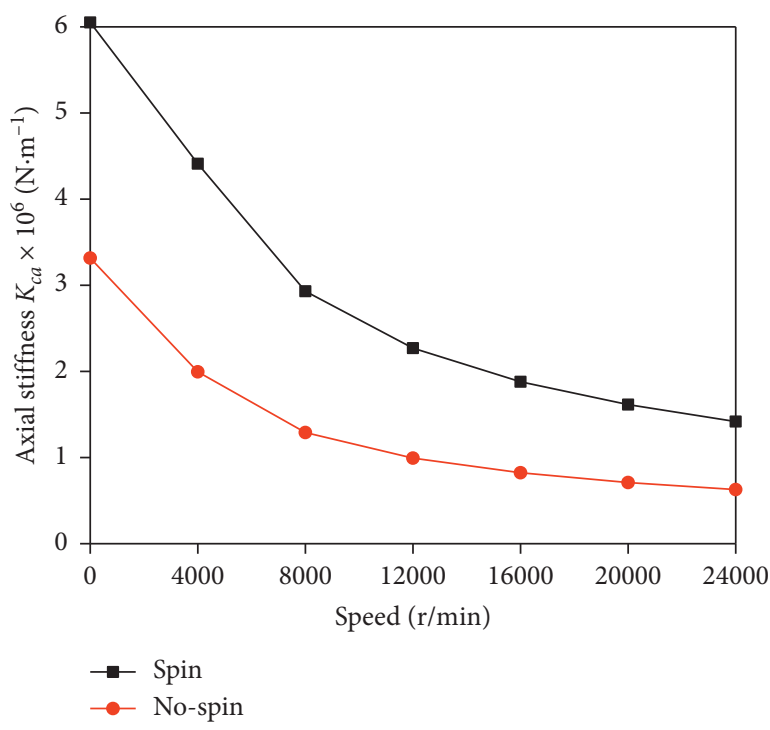

(b)

FIGURE 20: Continued. 


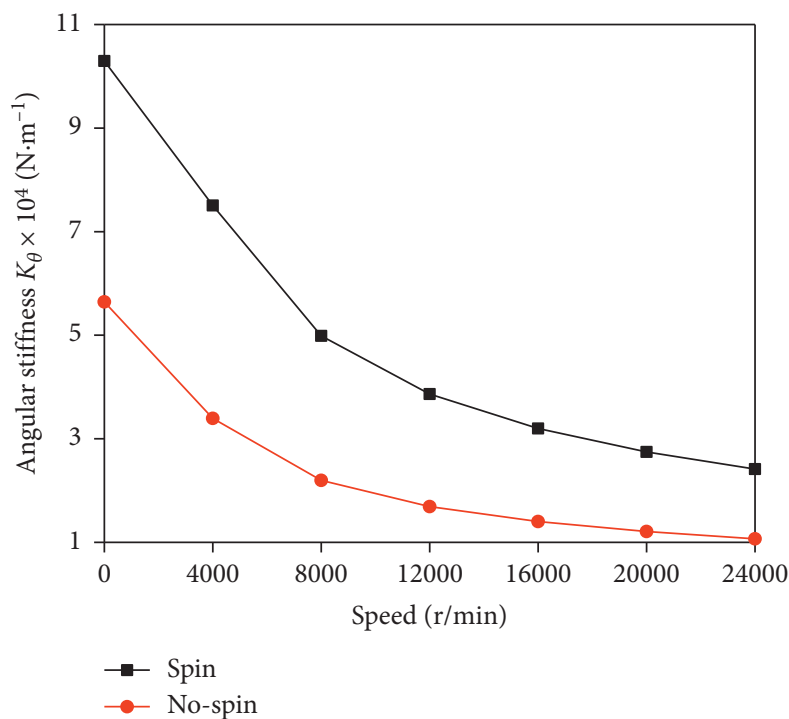

(c)

Figure 20: Influences of the speed and spin on the composite stiffness. (a) Radial stiffness. (b) Axial stiffness. (c) Angular stiffness.

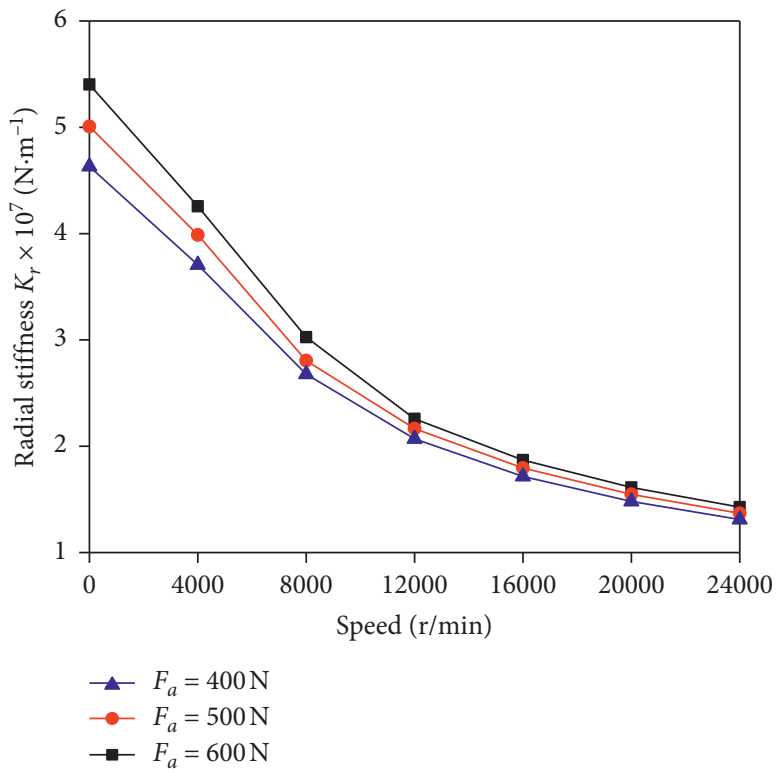

(a)

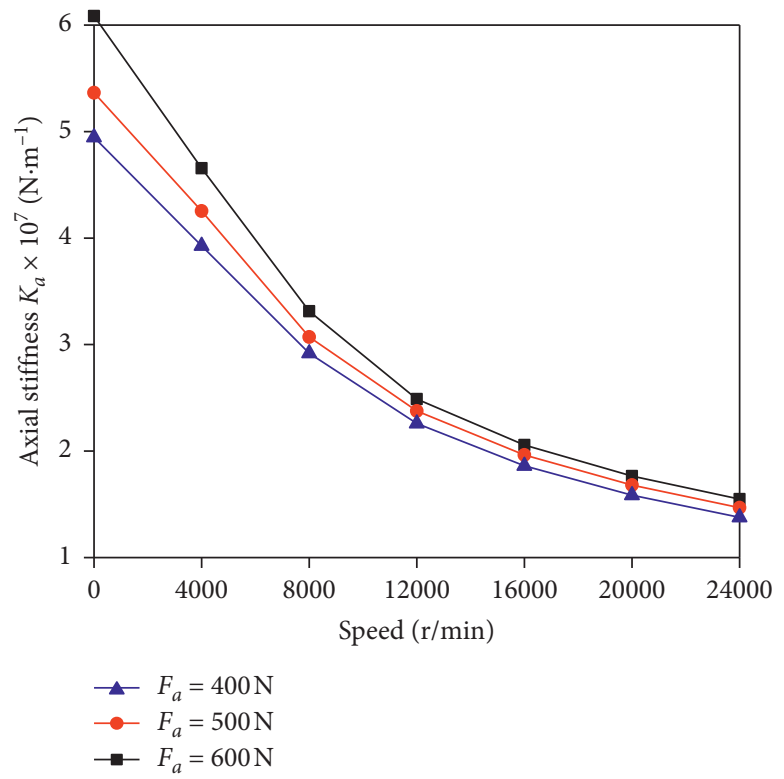

(b)

FIGURE 21: Continued. 


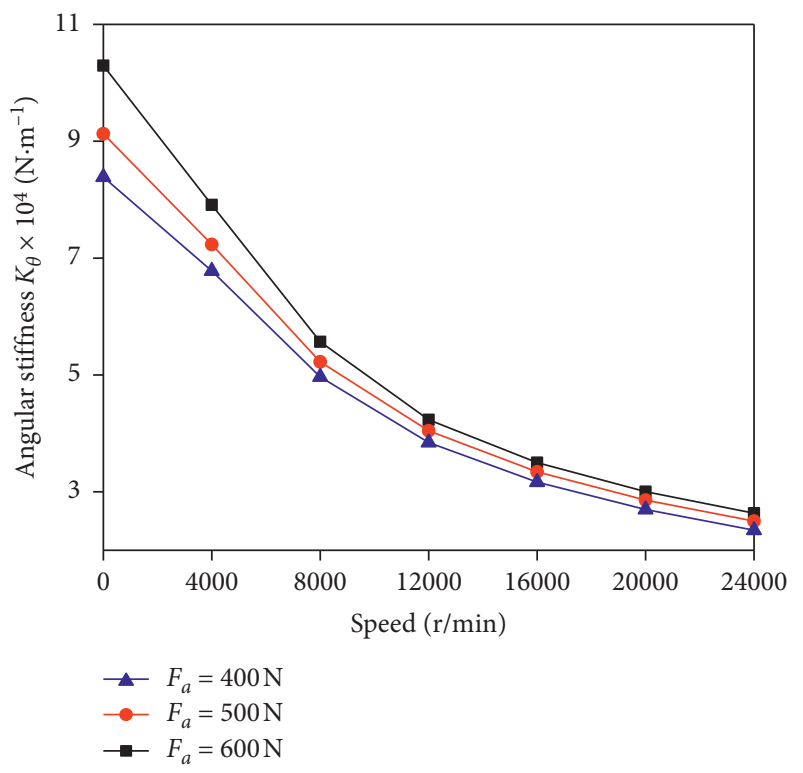

(c)

Figure 21: Influences of the speed and preload on the composite stiffness. (a) Radial stiffness. (b) Axial stiffness. (c) Angular stiffness.

occurs which changes under the action of the positioning preload, and the axial deformation increases with the increase in speed, resulting in the decrease of the axial stiffness.

\subsubsection{Influence of Deformation on Contact Stiffness.} Figure 19 shows the influence of deformation on the contact stiffness of the ball bearing, including no deformation, thermal deformation, centrifugal deformation, and combined deformation. It can be seen that the radial stiffness increases with the increase in speed; on the contrary, the axial and angular stiffness decrease gradually. In Figure 19(a), it can be known that when the speed is less than $8000 \mathrm{r} / \mathrm{min}$, the thermal deformation has great impact on the radial stiffness, and the stiffness considering the thermal deformation is higher than that considering the centrifugal deformation. When the speed is larger than $8000 \mathrm{r} / \mathrm{min}$, the radial stiffness is more sensitive to the centrifugal effect, and the stiffness considering the centrifugal deformation is higher. In Figures 19(b) and 19(c), it can be seen that the stiffness curve considering the centrifugal deformation is higher than that considering the thermal deformation when the speed is less than $8000 \mathrm{r} / \mathrm{min}$, while it is the opposite when the speed is higher than $8000 \mathrm{r} / \mathrm{min}$. This is mainly due to the fact that when the speed is lower than $8000 \mathrm{r} / \mathrm{min}$, the axial centrifugal deformation is smaller than the thermal deformation, and while the rotating speed is higher than $8000 \mathrm{r} / \mathrm{min}$, the thermal deformation is smaller, which results in the intersection of two curves as shown in Figures 19(b) and 19(c).

\subsection{Influence of Multiple Factors on the Composite Stiffness of Bearing}

5.5.1. Influence of Spinning Motion on Composite Stiffness. Figure 20 shows the influence of spin motion on the composite stiffness of the angular contact ball bearing. In
Figure 20, with the increase in speed, the composite stiffness (radial stiffness, axial stiffness, and angular stiffness) decreases. However, the composite stiffness increases under the influence of the spin motion. The reason for this is that both the film stiffness and the contact stiffness of the inner and outer rings increase with the spin motion, which results in the composite stiffness increasing.

5.5.2. Influence of Preload on the Composite Stiffness. In order to further study the influence of preload on the bearing stability, when the axial preload is $400 \mathrm{~N}, 500 \mathrm{~N}$, and $600 \mathrm{~N}$, respectively, the composite stiffness under the condition of spin motion is calculated, and the influence rule of axial preload on the composite stiffness is analyzed. Figure 21 shows the influence of preload on the composite stiffness. It can be seen that the composite stiffness increases gradually with the axial preload increasing. The reason is that both the film stiffness and the contact stiffness increase with the increase in axial preload, and the composite stiffness is composed of the contact stiffness and film stiffness in series. In addition, it can be seen that the influence of axial preload on the composite stiffness decreases with the increase in speed.

5.5.3. Effect of EHL on Composite Stiffness. Figure 22 shows the effect of EHL on the overall stiffness of ball bearings. From Figure 22, it can be seen that the radial composite stiffness considering the effect of EHL is larger than that without lubrication, while the axial and angular stiffness are smaller, and the trends change obviously at high speed. The main reason is that the radial contact stiffness under EHL effect is larger than that under no EHL, while the axial and angular stiffness are smaller; moreover, the magnitude of the contact stiffness is close to that of the film stiffness under this 


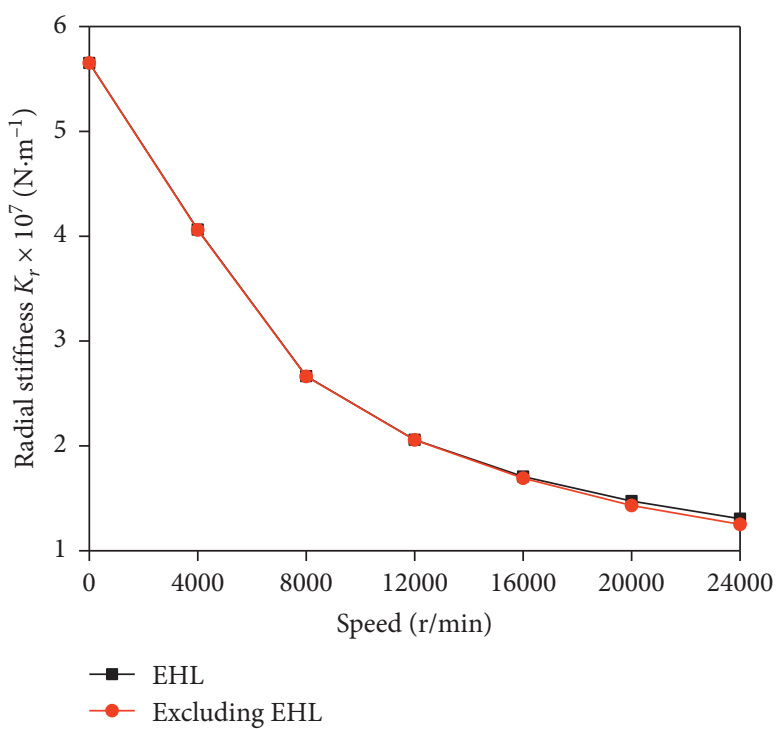

(a)

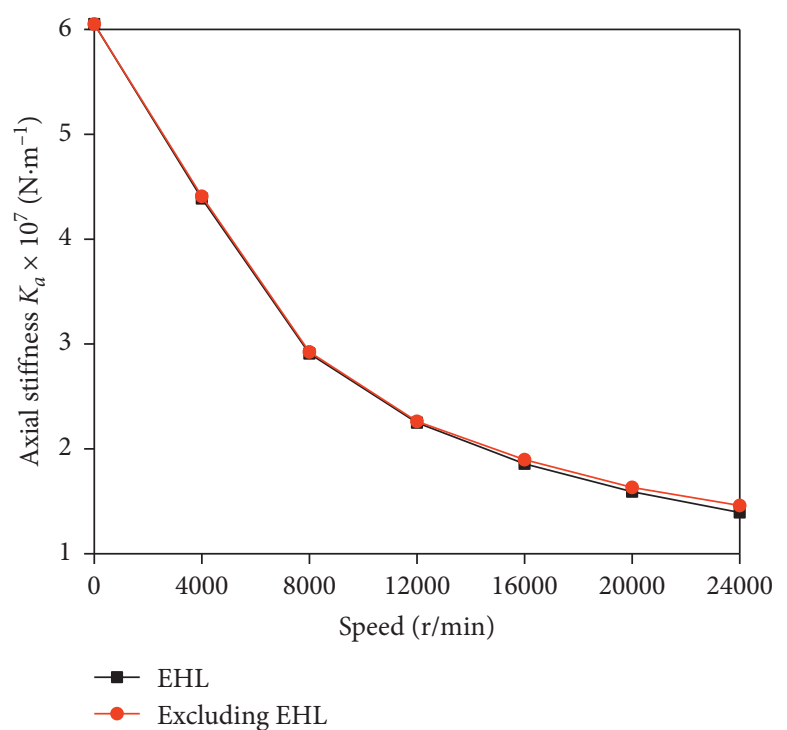

(b)

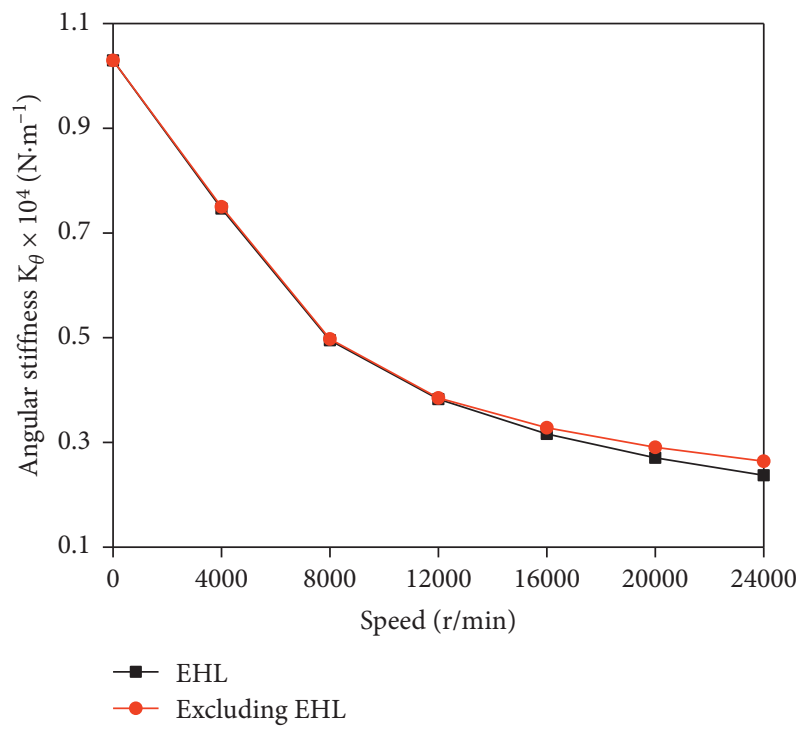

(c)

Figure 22: Influences of the speed and EHL on the composite stiffness. (a) Radial stiffness. (b) Axial stiffness. (c) Angular stiffness.

situation; therefore, when they are combined in series, the radial stiffness is larger, while the radial stiffness and angular stiffness are smaller.

5.5.4. Influence of Deformation on Composite Stiffness. In this section, the composite stiffness of the ball bearing is calculated under the conditions of no deformation, centrifugal deformation, thermal deformation, and integrated deformation, respectively, which is shown in Figure 23. In Figure 23(a), it can be seen that the influence of the deformation on the radial composite stiffness is more obvious with the increase in speed. Considering the thermal deformation and centrifugal deformation, respectively, the radial composite stiffness increases. At the same time, the effect of only considering the centrifugal deformation on the radial composite stiffness is greater than that only considering the thermal deformation. In Figures 23(b) and 23(c), the axial and angular composite stiffness decrease and the variation amplitude is the largest considering all deformation. According to the influence laws of each deformation on contact stiffness in Section 5.4.3 and the film stiffness model established in Section 4.1, it can be known that the effect of thermal deformation and centrifugal deformation on film stiffness is small. When the film stiffness is connected in series with contact stiffness, the influence of each deformation on composite stiffness is the same as that on contact stiffness, and the integrated deformation has the greatest influence on radial composite stiffness. In addition, the influence of 


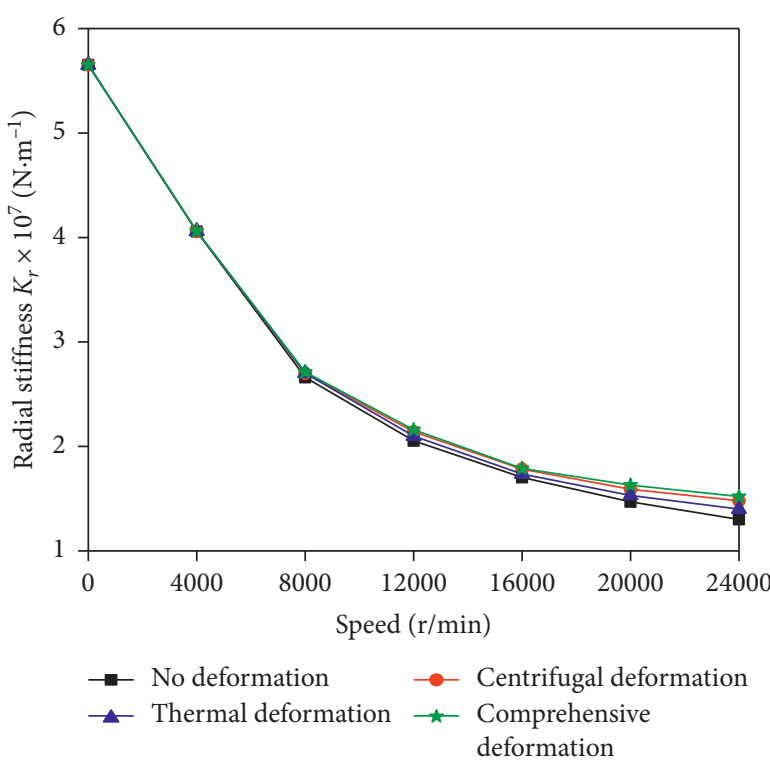

(a)

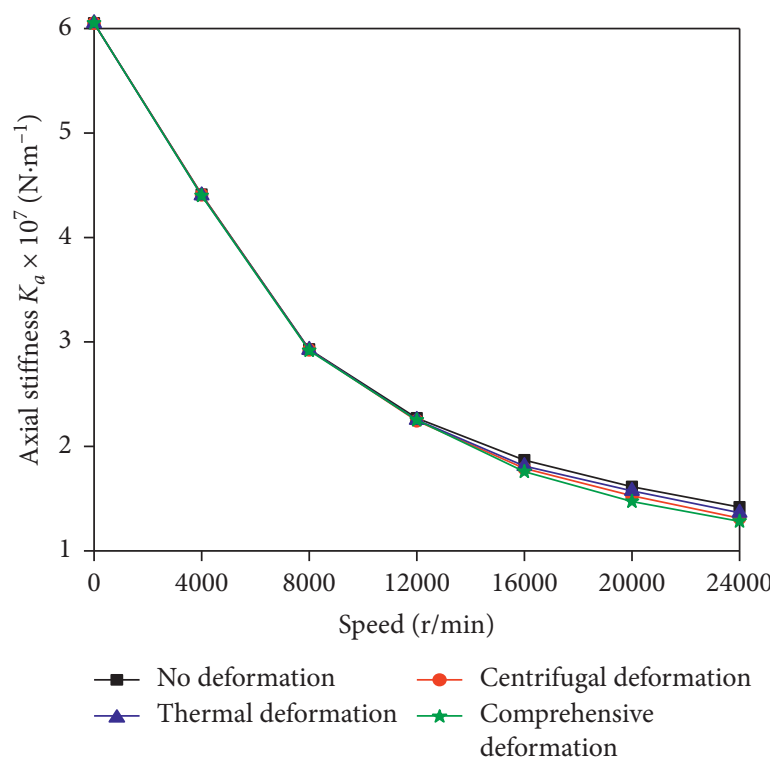

(b)

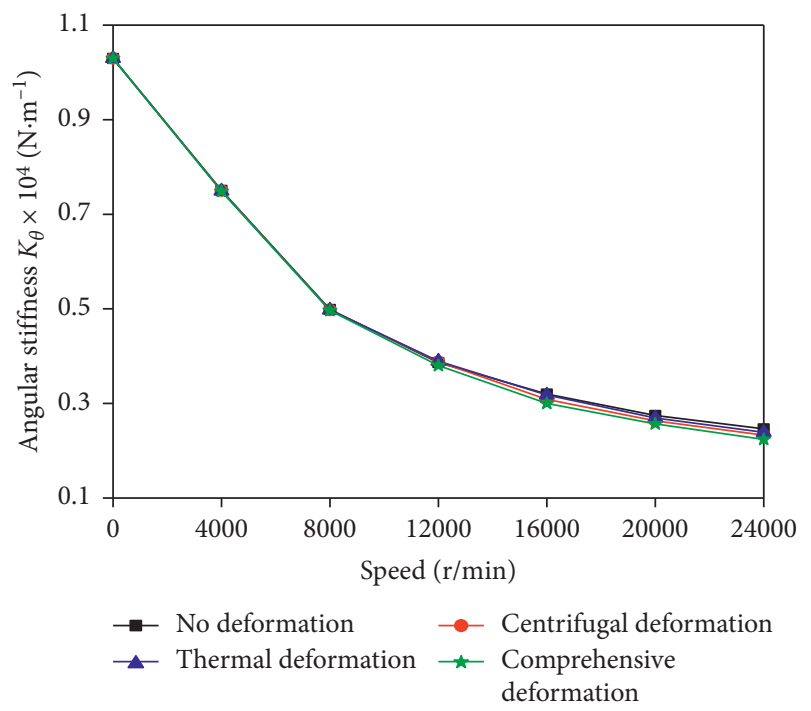

(c)

Figure 23: Influences of the speed and deformation on the composite stiffness. (a) Radial stiffness. (b) Axial stiffness. (c) Angular stiffness.

each deformation on the axial and angular composite stiffness is small.

\section{Conclusion}

Based on Jones' quasistatic model and EHL theory, considering the centrifugal deformation, thermal deformation, and EHL, the modified quasistatic model of the angular contact ball bearing is proposed. On the basis, a composite stiffness model of the ball bearing is presented by combining the contact stiffness with film stiffness considering the spinning motion in series. Moreover, the influences of various factors on the dynamic characteristic parameters and stiffness are studied in detail. The conclusions are as follows:

(1) The film stiffness of the bearing decreases with the increase in speed. The film stiffness considering the spin motion is greater than that without the spin. When the spin motion is considered, the composite stiffness increases.

(2) Considering the preload, the contact angles and loads of the inner and outer rings increase, and the contact stiffness and composite stiffness of the bearing also increase.

(3) Under the impact of EHL, the contact angles of the inner and outer rings decrease and the contact loads increase; the radial contact stiffness and composite stiffness increase, while the axial and angular contact stiffness and composite stiffness decrease.

(4) When the various deformation are considered, the bearing contact angles decrease and the contact loads increase, and the radial contact stiffness and 
composite stiffness increase, while the axial and angular contact stiffness and composite stiffness decrease.

\section{Nomenclature}

a: Thermal expansion coefficient

$A_{1}$ : $\quad$ Axial distances between the inner and outer ring groove curvature center

$A_{2}$ : $\quad$ Radial distances between the inner and outer ring groove curvature center

$\alpha$ : $\quad$ Contact angle after preloading

$\alpha_{i}$ : $\quad$ Inner ring contact angle

$\alpha_{o}$ : Outer ring contact angle

$\alpha_{p}$ : Lubricating oil viscosity-pressure coefficient

$\alpha_{0}$ : Original contact angle

$\alpha^{\prime}: \quad$ Contact angle before preloading

$\alpha^{(k+1)}$ : New contact angle after iteration

$\alpha^{(k)}$ : Contact angle before iteration

$D_{b}$ : Rolling element diameter

$D_{i}$ : $\quad$ Diameter of inner ring

$d$ : Inner diameter

$d_{m}$ : Bearing pitch diameter

$d_{T}: \quad$ Diameter of part

$\delta$ : $\quad$ Elastic deformation

$\delta_{a}^{\prime}$ : Displacement of the rolling element relative to the raceway

$\delta_{1}$ : $\quad$ Radial deformation of inner ring due to centrifugal effect

$\delta_{2}$ : $\quad$ Radial deformation caused by temperature rise

$\delta_{3}$ : $\quad$ Radial deformation of inner ring after bearing assembly

$\delta_{4}: \quad$ Initial interference

$\Delta \delta: \quad$ Displacement change of the center point of the ball under the action of EHL

$\Delta T: \quad$ Temperature rise

$E: \quad$ Equivalent elastic modulus

$E_{i}$ : $\quad$ Elastic modulus of inner ring

$F_{a}$ : Axial load

$F_{r}: \quad$ Radial load

$G$ : $\quad$ Material parameter

$\eta_{0}: \quad$ Initial dynamic viscosity

$h_{0}$ : Central film thickness

$\rho_{0}$ : $\quad$ Ambient density

$H_{\text {mins }}$ : Dimensionless minimum film thickness

$h_{\text {min }}$ : Minimum film thickness

$K$ : $\quad$ Contact deformation coefficient

$K_{a}: \quad$ Axial composite stiffness

$K_{\theta}: \quad$ Angular composite stiffness

$K_{c}: \quad$ Contact stiffness

$K_{\text {films }}:$ Film stiffness

$K_{n}$ : Equivalent load-deformation coefficient

$K_{r}: \quad$ Radial composite stiffness

$k$ : $\quad$ Ellipticity

$l$ : $\quad$ Effective contact lengths

$M_{g}$ : Gyroscopic moment

$P: \quad$ Assembly stress

$\rho$ : $\quad$ Material density

Q: $\quad$ Contact load $r$ : Raceway groove curvature radius

$r_{1}$ : Inner radius of inner ring

$r_{2}$ : Outer radius of inner ring

$U: \quad$ Dimensionless velocity parameter

$u$ : Thermal deformation

$u_{a}: \quad$ Axial deformation

$u_{r}$ : Radial deformation

$v$ : Poisson ratio

$\Omega_{s}: \quad$ Spinning angular velocity parameter

$Z: \quad$ The number of balls

b: $\quad$ Ball

$i$ : Inner raceway

$s: \quad$ Shaft

$h$ : Bearing housing

$j$ : $\quad$ Rolling element at angular location

$o$ : Outer raceway.

\section{Data Availability}

The data used to support the findings of this study are included in the Supplementary Materials.

\section{Conflicts of Interest}

The authors declare that they have no conflicts of interest.

\section{Acknowledgments}

This research was financially supported by the National Science Foundation of China (grant nos. 51465035 and 51965035).

\section{References}

[1] R. Ganesan, "Dynamic response and stability of a rotorsupport system with non-symmetric bearing clearances," Mechanism and Machine Theory, vol. 31, no. 6, pp. 781-798, 1996.

[2] A. B. Jones, "Ball motion and sliding friction in ball bearings," Journal of Basic Engineering, vol. 81, no. 1, pp. 1-12, 1959.

[3] T. A. Harris, Rolling Bearing Analysis, John Wiley \& Sons, New York, NY, USA, 1984.

[4] P. Gupta, Advanced Dynamics of Rolling Elements, Springer, New York, NY, USA, 1984.

[5] C. Li and Z. Sun, "Dynamic virtual simulation of deep groove ball bearings based on EHL theory," Chinese Journal of Aerodynamics, vol. 24, no. 4, pp. 951-956, 2009.

[6] D. Noel, M. Ritou, B. Furet, and S. L. Loch, "Complete analytical expression of the stiffness matrix of angular contact ball bearings," Journal of Tribology, vol. 135, no. 4, pp. 041101-041108, 2013.

[7] J. Liu, C. Tang, H. Wu, X. Zidana, and W. Linfenga, "An analytical calculation method of the load distribution and stiffness of an angular contact ball bearing," Mechanism and Machine Theory, vol. 142, Article ID 103597, 2019.

[8] L. Bizarre, F. Nonato, and K. L. Cavalca, "Formulation of five degrees of freedom ball bearing model accounting for the nonlinear stiffness and damping of elastohydrodynamic point contacts," Mechanism and Machine Theory, vol. 124, pp. 179-196, 2018.

[9] K. Guo, S. H. Yuan, Y. Y. Zhang et al., "Study on the calculation method of ball bearing mechanical characteristics 
considering elastohydrodynamic lubrication with spinning," Journal of Mechanical Engineering, vol. 49, no. 15, pp. 62-67, 2013.

[10] C. Lei, F. Li, J. Guo et al., "Oil film stiffness analysis of rolling bearing based on multi parameter coupling," Vibration and Shock, vol. 37, no. 10, pp. 225-232, 2018.

[11] Y. Zhang, X. h. Li, J. Hong, K. Yan, and S. Li, "Uneven heat generation and thermal performance of spindle bearings," Tribology International, vol. 126, pp. 324-335, 2018.

[12] J. Liu, "A dynamic modelling method of a rotor-roller bearing-housing system with a localized fault including the additional excitation zone," Journal of Sound and Vibration, vol. 469, p. 115144, 2020.

[13] J. Liu and Y. Shao, "Dynamic modeling for rigid rotor bearing systems with a localized defect considering additional deformations at the sharp edges," Journal of Sound and Vibration, vol. 398, pp. 84-102, 2017.

[14] C. Lei, F. Li, B. Gong, and X. Jia, "An integrated model to characterize comprehensive stiffness of angular contact ball bearings," Mathematical Problems in Engineering, vol. 2020, Article ID 4951828, 12 pages, 2020.

[15] S. H. Wang and H. H. Zhang, "Combined effects of thermal and non-Newtonian character of lubricant on pressure, film profile, temperature rise, and shear stress in E.H.L." Journal of Tribology, vol. 109, no. 4, pp. 666-670, 1987.

[16] R. Kumar, M. S. Azam, S. K. Ghosh, and H. Khan, "Thermoelastohydrodynamic lubrication simulation of the Rayleigh step bearing using the progressive mesh densification method," Simulation, vol. 95, no. 5, pp. 395-410, 2019.

[17] R. Kumar, M. S. Azam, and S. K. Ghosh, "Influence of stochastic roughness on performance of a Rayleigh step bearing operating under thermo-elastohydrodynamic lubrication considering shear flow factor," Tribology International, vol. 134, pp. 264-280, 2019.

[18] T. Xu, G. Xu, Q. Zhang et al., "A preload analytical method for ball bearings utilising bearing skidding criterion," Tribology International, vol. 67, pp. 44-50, 2013.

[19] B. Wang, X. Mei, C. Hu et al., "Dynamic characteristics analysis of preloaded high-speed angular contact ball bearings," Bearing, vol. 2010, no. 5, pp. 1-4, 2010.

[20] A. Gunduz, J. T. Dreyer, and R. Singh, "Effect of bearing preloads on the modal characteristics of a shaft-bearing assembly: experiments on double row angular contact ball bearings," Mechanical Systems \& Signal Processing, vol. 31, pp. 176-195, 2012.

[21] B. Li, J. B. Zhang, and L. S. Jiang, "A research of shaft modal simulation method based on the preloaded angular contact ball bearing," Applied Mechanics and Materials, vol. 389, pp. 364-370, 2013.

[22] J. Kraus, J. J. Blech, and S. G. Braun, "In situ determination of rolling bearing stiffness and damping by modal analysis," Journal of Vibration and Acoustics, vol. 109, no. 3, pp. 235240, 1987.

[23] X. Yun, X. Mei, G. Jiang et al., "Dynamic stiffness analysis and test method of high speed spindle angular contact ball bearing," Vibration, Test and Diagnosis, vol. 39, no. 4, pp. 892-897, 912, 2019.

[24] S. Li, X. Chen, G. Zhang et al., "Analyses of dynamic supporting stiffness about spindle bearings at extra high-speed in electric spindles," Chinese Journal of Mechanical Engineering, vol. 42, no. 11, pp. 60-65, 2006.

[25] X. Li, R. Chen, D. Shang et al., "Dynamic response of maneuvering flight friction rotor with variable stiffness bearing,"
Journal of Harbin University of Technology, vol. 52, no. 1, pp. 1-7, 2020.

[26] W. Xiong, Y. Zhou, Z. Zhao et al., "Quantitative research on the permissible tilt angle of high-speed angular contact ball bearings," Journal of Mechanical Engineering, vol. 51, no. 23, pp. 46-52, 2015.

[27] J. Okamoto, Design and Calculation of Ball Bearing, China Machine Press, Beijing, China, 2004.

[28] M. Wu, W. Wu, J. Hu et al., "Oil film stiffness calculation of high speed angular contact ball bearings considering spinning," Vibration and Shock, vol. 33, no. 10, pp. 38-42, 2014.

[29] H. Ping, Numerical Calculation Method of Dynamic Fluid Dynamic Lubrication, Tsinghua University Press, Beijing, China, 2013.

[30] B. Chen, J. Chen, M. Dong et al., "Modeling method analysis for oil film stiffness calculation based on point contact TEHL EHL," Lubrication Engineering, vol. 38, no. 8, pp. 70-75, 2013.

[31] B. Wang, C. Hu, Z. Wu et al., "Effect of preloading on dynamic stiffness of high speed angular contact ball bearing," Journal of Lanzhou University of Technology, vol. 35, no. 2, pp. 29-32, 2009.

[32] Y. Zhang, Study on Thermal Characteristics of Multi-Parameter Affected of High-Speed Motorized Spindle, Lanzhou University of Technology, Lanzhou, China, 2019. 